DEPARTMENT OF THE INTERIOR

U.S. GEOLOGICAL SURVEY

\title{
Palynological Data from the Fort Union and Wasatch Formations, Powder River Basin, Wyoning and Montana
}

\author{
by \\ D.T. Pockna11 \\ New Zealand Geological Survey, Lower Hutt, New Zealand
}

Open-File Report 86-117

This report is preliminary and has not been reviewed for conformity with U.S. Geological Survey editorial standards and stratigraphic nomenclature. 
CONTENTS

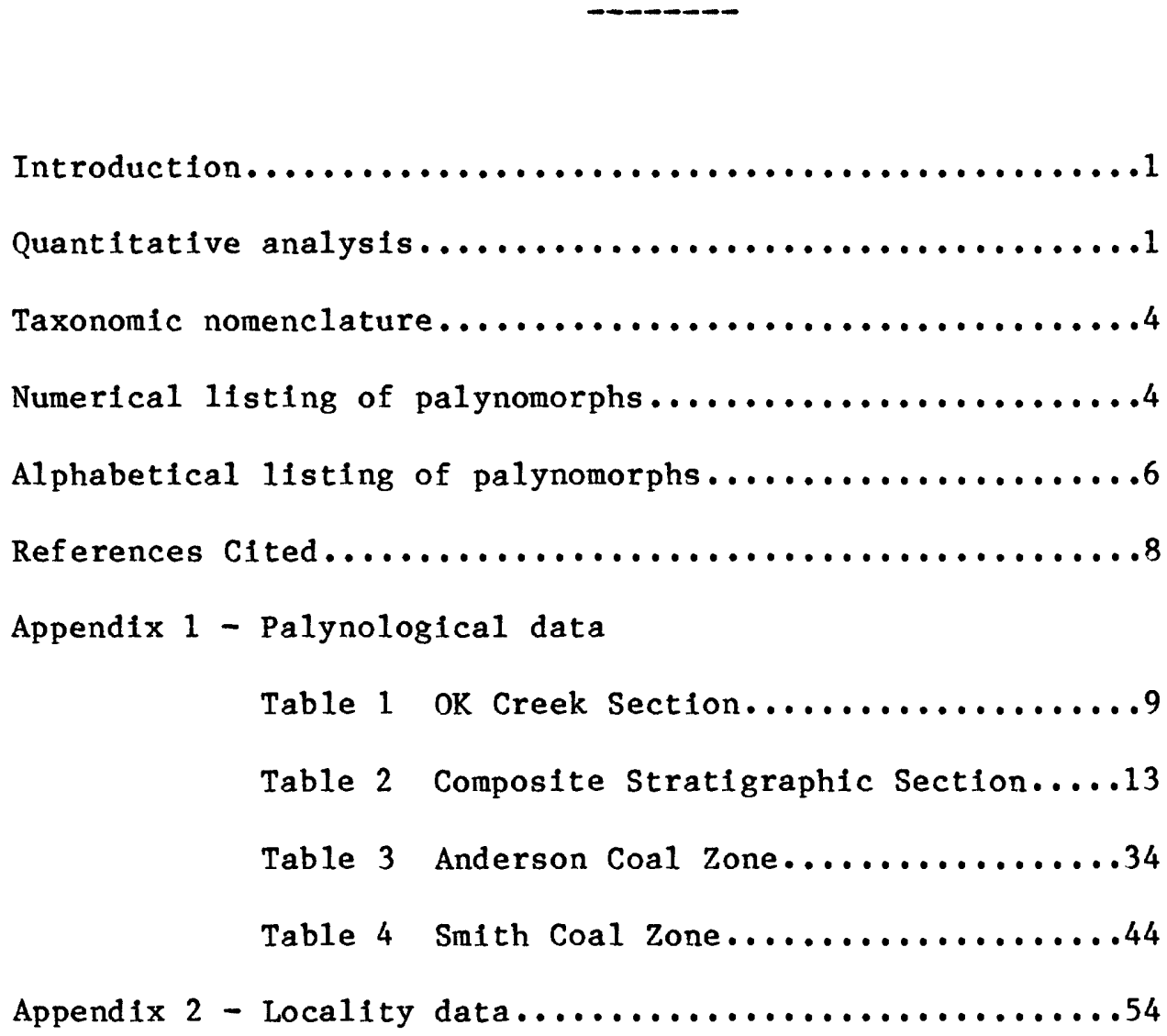

Text Figures

Figure 1: Locality map..................

Figure 2: Composite stratigraphic section......3 


\section{INTRODUCTION}

The palynological data presented in this report result from a biostratigraphic and paleoecologic study of the Fort Union and Wasatch Formations in the northern Powder River Basin, Wyoming and Montana. This work has been the subject of an assignment by the author while on leave from the New Zealand Geological Survey, Lower Hutt, New Zealand and as a Visiting Scientist at the U.S. Geological Survey, Denver, Colorado.

Samples for palynological examination were obtained from core and surface sections near Arvada, Wyoming, and Broadus, Montana (see Fig. 1). Cores were placed in stratigraphic context by Flores et al. (1982). Fig. 2 is a composite stratigraphic section of the Tongue River Member of the Fort Union Formation and the lowermost Wasatch Formation. On this figure are marked the major coal beds and the position of samples collected for a stratigraphic analysis (prefixed with a USGS Paleobotany "D" number). Coal bed nomenclature follows Olive (1957), Flores et al. (1982), and McLellan (1981; oral communication, 1985).

The data are presented in two appendices: in Appendix 1 all the palynological data are tabulated, and in Appendix 2 the locality data for all samples are presented.

The data obtained from the biostratigraphic samples are presented in Tables 1 and 2 of Appendix 1 . A more detailed analysis was made of the Anderson and Smith coals in several cores and these data are recorded in Tables 3 and 4 of Appendix 1, respectively.

\section{QUANTITATIVE ANALYSIS}

Most samples examined contained a rich and well preserved palynomorph assemblage. Assemblages from the Tongue River Member were almost without exception dominated by pollen of the Taxodiaceae and Cupressaceae familles. This dominance tends to mask the more stratigraphically and paleoecologically important elements in the assemblages and for this reason are excluded from many percentage calculations. In several samples additional palynomorph counts were required to obtain a statistically reliable base for percentage calculations, a minimum count of 100 palynomorphs. These additional counts are recorded in parentheses in Table 2 of Appendix 1 (e.g., 3(7)). In a11 tables a " + " indicates the palynomorph was recorded after the count was completed. A "-" indicates the absence of the palynomorph.

Al1 slides are held in the Denver laboratory of the U.S. Geological Survey. They may be accessed by their " $D$ " numbers. 


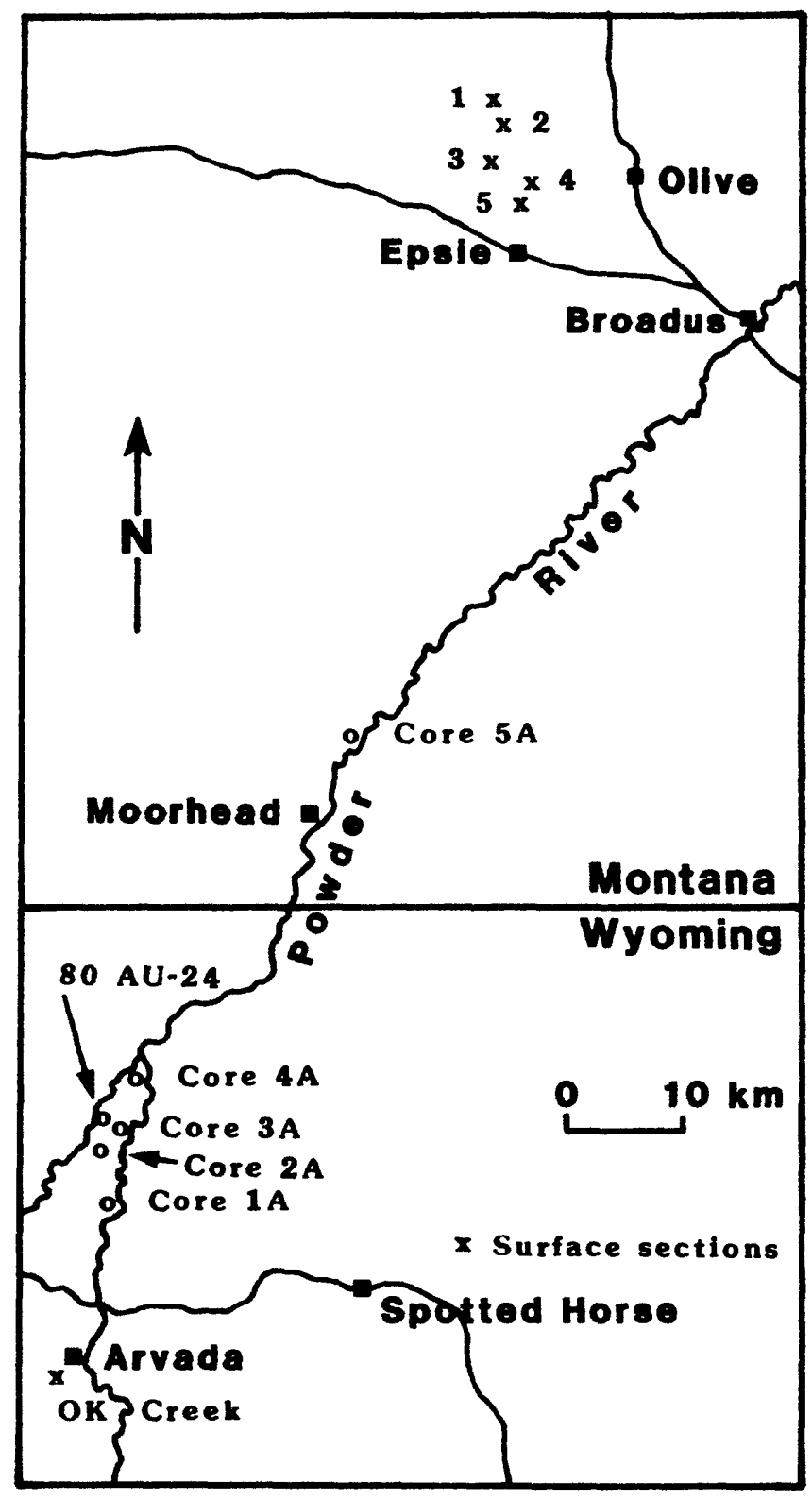

Fig. 1. Locality of cores and surface sections studied in the Powder River Basin. Surface localities are:

1. O'Dell coal (D6768). 2. Below O'Dell coal (D6767).

3. Mackin-Walker coal (D6765\&D6764). 4. Local 2 coal (D6766). 5. Sawyer coal (D6763\&D6762). 


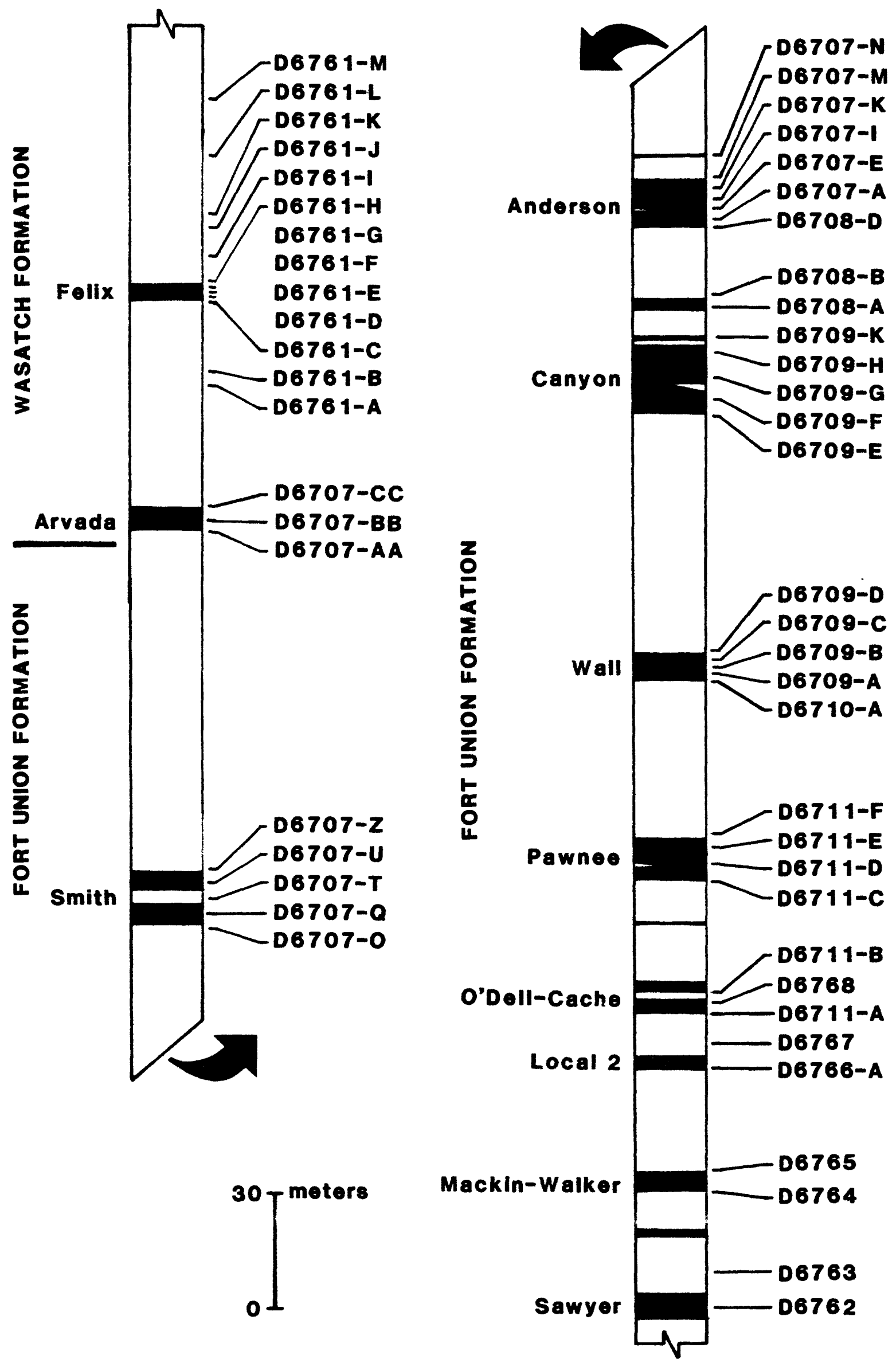

Fig. 2. Composite stratigraphic section showing distribution of coal beds and sampling horizons in the Fort Union and Wasatch Formations, Powder River Basin. 


\section{TAXONOMIC NOMENCLATURE}

For ease of tabulation, all palynomorphs have been assigned a number. The numbers appear along the horizontal axes of all tables. Both numerical and alphabetical listings are provided.

No new taxonomic names are used in this report. Some taxa which are undescribed at present have been assigned either to genera or to a morphological type (e.g., Tricolporate A.), when no described genus is considered suitable. A variety of tricolpate and tricolporate taxa were recorded that could not be assigned to existing species or genera; these have been included in the category "Tricolpate/tricolporate unknown". Taxodiaceae and Cupressaceae types have been recorded collectively under their family affinity because of the difficulty in distinguishing between them. Although several bisaccate conifer pollen types were noted, they are not subdivided into fossil generic taxa; they have been recorded as "bisaccates".

\section{NUMERICAL LISTING OF PALYROMORPHS}

\section{Taxodiaceae - Cupressaceae}

2. Bisaccates

3. Caryapollenites veripites (Wilson \& Webster) Nichols \& Ott

4. C. imparalis Nichols \& Ott

5. C. Inelegans Nichols \& Ott

6. C. wodehousei Nichols \& Ott

7. Momipites wyomingensis Nichols \& Ott

8. M. ventifluminis Nichols \& Ott

9. M. annellus Nichols \& Ott

10. M. amplus (Leffingwe11) Nichols

11. M. waltmanensis Nichols \& Ott

12. Ulmipollenites krempii/tricostatus

13. Alnipollenites verus (Potonie) Potonie

14. A. trina (Stanley) Norton \& Hall

15. Alnus scoticus Simpson

16. Tilia tetraforaminipites Wodehouse

17. Triporopollenites spp.

18. Quercus explanata Anderson

19. Nyssa ingentipollinia Traverse

20. Tricolporate A. 
21. Tricolporate B.

22. Cupuliferoipollenites spp.

23. Triatriopollenites granulatus (Simpson) Leffingwell

24. Polyatriopollenites vermontensis (Traverse) Frederiksen

25. Paraalnipollenites $\mathrm{cf} . \underline{\mathrm{P}}$. confusus Hills \& Wallace

26. Pistillipollenites mcgregorii Rouse

27. Insulapollenites rugulatus Leffingwell

28. Syncolporites cf. . minimus Leffingwell

29. Tricolpate (fine reticulum)

30. Tricolpites hians Stanley

31. Tricolpate A.

32. Tricolpate B.

33. Tricolpate C.

34. Tricolpites anguloluminosus Anderson

35. Erdtmanipollis pachysandroides Krutzsch

36. Periporopollenites sp.

37. Malvacipollis spp.

38. Liliacidites spp.

39. Arecipites cf. A. tenuiexinous Leffingwell

40. Pandanildites radicus Leffingwell

41. Ericipites $s p$.

42. Corollina sp.

43. Ephedripites spp.

44. Cycadopites spp.

45. ?Araucariacites sp.

46. Tricolpites sp. cf. Eucommia

47. Aesculildites sp.

48. Aesculiidites circumstriatus (Falrchild) Elsik

49. Monosulcites spp.

50. Sparganium cf. S. globipites Wilson \& Webster

51. Dyadonipites reticulatus Tschudy

52. Platycarya platycaryoides (Roche) Frederiksen \& Christopher

53. Aquilapollenites spinulosus Funkhouser

54. Ailanthipites cf. A. berryi Wodehouse

55. Intratriporopollenites sp.

56. Proteacidites cf. $\underline{\text { P }}$ thalmanni Anderson 
57. Tricolpate/tricolporate unknown

58. Leiotriletes spp.

59. Gleichenilites sp.

60. Stereisporites spp.

61. Reticuloidosporites pseudomurii Elsik

62. Laevigatosporites spp.

63. Lycopodiumsporites sp.

64. Baculatisporites sp.

65. Zlivisporis novomexicanum (Anderson) Leffingwel1

66. Cicatricosisporites sp.

\section{ALPHABETICAL LISTING OF PALYNOMORPHS}

Aesculiidites sp. $\quad 47$

Aesculiidites circumstriatus (Fairchild) E1sik 48

Ailanthipites cf. A. berryi Wodehouse

Alnipollenites trina (Stanley) Norton \& Hall 14

A. verus (Potonié) Potonié 13

Alnus scoticus Simpson $\quad 15$

Aquilapollenites spinulosus Funkhouser

$\begin{array}{ll}\text { ?Araucariacites sp. } & 45\end{array}$

Arecipites cf. A. tenuiexinous Leffingwell

Baculatisporites sp. $\quad 64$

Bisaccates 2

Caryapollenites Imparalis Nichols \& Ott 4

C. inelegans Nichols \& Ott 5

C. veripites (Wilson \& Webster) Nichols \& ott 3

C. wodehousei Nichols \& 0tt 6

Cicatricosisporites sp. $\quad 66$

$\begin{array}{ll}\text { Corollina sp. } & 42\end{array}$

$\begin{array}{ll}\text { Cupuliferoipollenites spp. } & 22\end{array}$

$\begin{array}{ll}\text { Cycadopites spp. } & 44\end{array}$

Dyadonipites reticulatus Tschudy

Ephedripites spp. $\quad 43$ 
Erdtmanipollis pachysandroides Krutzsch

$\begin{array}{ll}\text { Ericipites sp. } & 41\end{array}$

Gleichenilitites sp. $\quad 59$

Insulapollenites rugulatus Leffingwell $\quad 27$

Intratriporopollenites sp. $\quad 55$

$\begin{array}{ll}\text { Laevigatosporites spp. } & 62\end{array}$

Leiotriletes spp. $\quad 58$

\begin{tabular}{ll} 
Liliacidites spp. & 38 \\
\hline
\end{tabular}

\begin{tabular}{ll} 
Lycopodiumsporites & 63 \\
\hline
\end{tabular}

Malvacipollis spp. $\quad 37$

Momipites amplus (Leffingwe11) Nichols $\quad 10$

M. annellus Nichols \& Ott 9

M. ventifluminis Nichols \& Ott

M. waltmanensis Nichols \& Ott 11

M. wyomingensis Nichols \& Ott 7

\begin{tabular}{ll} 
Monosulcites spp. & 49 \\
\hline
\end{tabular}

Nyssa ingentipollinia Traverse 19

Pandaniidites radicus Leffingwell $\quad 40$

Paraalnipollenites cf. P. confusus Hills \& Wallace 25

Periporopollenites sp. $\quad 36$

Pistillipollenites mcgregorii Rouse $\quad 26$

Platycarya platycaryoides (Roche) Frederiksen \& Christopher 52

Polyatriopollenites vermontensis (Traverse) Frederiksen $\quad 24$

Proteacidites cf. $\underline{P}$ - thalmanni Anderson $\quad 56$

$\begin{array}{ll}\text { Quercus explanata Anderson } & 18\end{array}$

Reticuloidosporites pseudomurii E1sik

Sparganium cf. S. globipites Wilson \& Webster $\quad 50$

$\begin{array}{ll}\text { Stereisporites spp. } & 60\end{array}$

Syncolporites cf. S. minimus Leffingwell 28

Taxodiaceae - Cupressaceae 1

Tilia tetraforaminipites Wodehouse $\quad 16$

Triatriopollenites granulatus (Simpson) Leffingwe11 23

$\begin{array}{ll}\text { Tricolpate (fine reticulum) } & 29\end{array}$

Tricolpate A. $\quad 31$

Tricolpate B. $\quad 32$

$\begin{array}{ll}\text { Tricolpate C. } & 33\end{array}$ 
Tricolpate/tricolporate unknown

Tricolpites anguloluminosus Anderson $\quad 34$

$\begin{array}{ll}\text { T. hians Stanley } & 30\end{array}$

T. sp. cf. Eucommia $\quad 46$

Tricolporate A. 20

$\begin{array}{ll}\text { Tricolporate B. } & 21\end{array}$

$\begin{array}{ll}\text { Triporopollenites spp. } & 17\end{array}$

Ulmipollenites krempil/tricostatus 12

Zlivisporis novomexicanum (Anderson) Leffingwell 65

\section{REFERENCES CITED}

Flores, R.M., Toth, J.C., and Moore, T.A., 1982, Use of geophysical logs in recognizing depositional environments in the Tongue River Member of the Fort Union Formation, Powder River area, Wyoming and Montana. U.S. Geological Survey Open-File Report 82-576, 40 p.

McLellan, M.W., 1981, Geologic map and coal resources of the Leslie Creek Quadrangle, Powder River County, Montana. U.S. Geological Survey Miscellaneous Field Studies, Map MF-1296.

0live, W.W., 1957, The Spotted Horse Coalfield, Sheridan and Campbel1 Counties, Wyoming. U.S. Geological Survey Bulletin 1050, 83 p. 


\section{APPENDIX 1}

TABLE 1

PALYNOMORPHS IN THE OR CREER SECTION

\section{USGS Paleobotany}

locality no.

Palynomorph number

1. 2. 3. 4. 5. $7 \cdot$ 8. $9.412 .13 \cdot 14$.

\begin{tabular}{|c|c|c|c|c|c|c|c|c|c|c|}
\hline D6761-M & 5 & + & 3 & 4 & - & 3 & - & + & 47 & 3 \\
\hline D6761-L & 48 & 4 & 1 & 9 & 1 & 3 & - & - & 13 & + \\
\hline D6761-K & 31 & 3 & + & - & + & - & - & + & 4 & 10 \\
\hline D6 $761-J$ & 183 & + & 4 & 2 & 3 & 2 & + & - & 3 & + \\
\hline D6761-I & 188 & 3 & + & 3 & + & 1 & + & - & 12 & 3 \\
\hline D6 761-H & 129 & 2 & 1 & + & 1 & + & - & + & 70 & 2 \\
\hline $6761-G$ & - & + & + & 2 & 1 & 1 & + & + & 100 & 4 \\
\hline
\end{tabular}

D6761-F

9

D6761-

58

D6761-D

161

$+$

$+$

2

$+$

D6761-

361

1

1

$2+$

$4++$

33

D6761-

587

$+$

$$
1
$$

1

$-$

$1+$

$-$

$4 \quad 57$

D6761-A 148

11

12

$2+$

$4++$ 
TABLE 1

15. 16. 17. 19. 22. 24. 25. 26. 29. 30. 31. 34.

\begin{tabular}{|c|c|c|c|c|c|c|c|c|c|c|c|c|}
\hline D6761-M & 2 & 1 & 11 & - & - & - & + & 6 & 1 & 3 & - & - \\
\hline D6761-L & - & 1 & 6 & - & - & - & - & 9 & 3 & 5 & - & - \\
\hline D6761-K & + & - & + & - & + & - & - & 6 & - & 6 & - & - \\
\hline D6761-J & - & 1 & 3 & - & 3 & - & - & 4 & 2 & 37 & - & - \\
\hline D6761-I & - & 3 & 2 & + & - & + & - & 19 & 10 & 23 & - & 1 \\
\hline D6761-H & - & 2 & 1 & 1 & - & - & - & + & 2 & 4 & - & - \\
\hline D6761-G & - & - & 3 & - & - & + & - & 1 & 5 & + & - & + \\
\hline D6761-F & - & - & 4 & - & - & - & - & - & 6 & - & - & 12 \\
\hline D6761-E & - & - & 50 & - & - & - & - & - & - & 4 & - & + \\
\hline D6761-D & - & + & + & - & - & - & - & 1 & - & 35 & 1 & - \\
\hline D6761-C & - & 1 & 1 & 1 & 2 & - & - & 5 & - & 58 & - & + \\
\hline D6761-B & - & + & + & + & 2 & + & + & + & 1 & 100 & - & - \\
\hline D $6761-A$ & - & + & + & + & 2 & + & + & 21 & 2 & 24 & 7 & + \\
\hline
\end{tabular}


TABLE 1

35. 36. 37. 38. 40. 43. 44. 46. 50. 52. 54. 55.

\begin{tabular}{|c|c|c|c|c|c|c|c|c|c|c|c|c|}
\hline D6761-M & - & - & - & - & 4 & - & - & & 6 & 100 & - & + \\
\hline D6761-L & - & - & - & - & 5 & + & - & + & 47 & 50 & - & + \\
\hline D6761-K & - & - & - & + & + & - & - & - & 36 & 200 & - & 8 \\
\hline D6761-J & + & + & - & 2 & 5 & - & - & + & 3 & 50 & 4 & 2 \\
\hline D $6761-I$ & + & + & + & - & 2 & + & - & 3 & 4 & 5 & - & + \\
\hline D6761-H & - & - & - & + & - & - & - & + & 15 & 100 & - & + \\
\hline D6761-G & - & - & - & - & + & - & + & 1 & 7 & 78 & - & 2 \\
\hline D6761-F & - & - & + & - & - & - & - & 1 & - & - & - & - \\
\hline D6761-E & - & - & - & - & - & - & - & - & - & 1 & - & - \\
\hline D6761-D & + & + & + & + & 2 & - & - & - & 1 & 33 & - & 28 \\
\hline D6761-C & - & - & 2 & - & - & - & + & - & - & 32 & - & 1 \\
\hline D6761-B & + & + & - & - & 2 & - & - & - & 1 & 28 & - & + \\
\hline D6761-A & - & - & - & - & + & - & + & 1 & 7 & 34 & + & 9 \\
\hline
\end{tabular}


TABLE 1

57. 58. 59.60.61.62.64.65.66.

\begin{tabular}{|c|c|c|c|c|c|c|c|c|c|}
\hline D6761-M & 6 & - & + & - & - & 11 & - & - & - \\
\hline D6761-L & 2 & 3 & - & + & - & 8 & - & + & + \\
\hline D6761-K & 3 & 2 & - & - & - & 30 & - & - & - \\
\hline D6761-J & 33 & 15 & - & 2 & - & 97 & + & 2 & + \\
\hline D6761-I & 7 & 6 & - & - & + & 1 & + & - & - \\
\hline D6761-H & - & 1 & + & 1 & - & 8 & + & - & - \\
\hline D6761-G & - & 1 & - & - & - & 15 & - & - & - \\
\hline D6761-F & 24 & 2 & - & - & - & 13 & - & - & - \\
\hline D6761-E & 24 & - & - & 19 & - & - & - & - & - \\
\hline D6 761-D & 4 & + & + & + & + & 8 & - & - & - \\
\hline D6761-C & 4 & 2 & + & 1 & + & 25 & - & - & - \\
\hline D6761-B & 4 & 2 & - & - & - & 4 & - & - & - \\
\hline D $6761-A$ & 2 & 2 & + & + & - & 19 & - & - & + \\
\hline
\end{tabular}


TABLE 2

PALYNOMORPHS IN THE COMPOSITE STRATIGRAPHIC SECTION

USGS Paleobotany

Locality no.

\section{Palynomorph number}

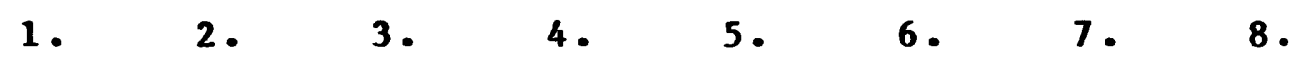

$\begin{array}{lrrrrrrr}\text { D6707-CC } & 200 & 7(31) & 5(5) & - & - & - & (1) \\ \text { D6707-B B } & 100 & 31 & 28 & - & 11 & - & +\end{array}$ -

$\begin{array}{lllllll}\mathrm{D} 6707-\mathrm{N} & 200 & 2(13) & 4(9) & 4(26) & (4) & (1)\end{array}$

D6707-M $200+2(13) 4(10) 1(1)+3(9)+$

D $6707-\mathrm{K} 200 \quad 5(23) \quad 1(3) \quad 10(11)-(1) 11(10)$

D6707-I $2002(17) \quad 4(9) \quad 2(11) 2(2)+(5) 10(10)+$

$\begin{array}{llllllll}\text { D } 6707-E & 200 & 8 & 20 & 26 & 9 & 7 & 25\end{array}$

D $6707-\mathrm{A} \quad 200 \quad 5(13) \quad 3(17) \quad 8(12) \quad 1(5)+(2) \quad 9(17)+(2)$

continued on p. 14 


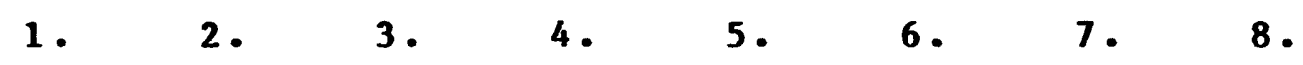

\begin{tabular}{|c|c|c|c|c|c|c|c|c|}
\hline D6708-D & 200 & $9(25)$ & $7(21)$ & $6(13)$ & (4) & $+(2)$ & $8(22)$ & $+(4)$ \\
\hline D6708-B & 200 & $1(5)$ & $+(5)$ & $+(3)$ & 2 & - & $+(14)$ & + \\
\hline D6708-A & 200 & 1 & + & 24 & 6 & - & 32 & 2 \\
\hline D6709-K & 200 & $+(2)$ & $+(5)$ & $4(4)$ & + & $+(1)$ & $2(7)$ & $+(2)$ \\
\hline D6709-J & + & - & - & + & + & - & + & - \\
\hline D6709-I & + & - & - & + & + & - & - & - \\
\hline D6709-H & 100 & 7 & + & 16 & 1 & - & 29 & + \\
\hline D6709-G & 200 & 4 & 3 & 8 & 1 & - & 21 & - \\
\hline D6709-F & 200 & 4 & 2 & 3 & + & + & 12 & + \\
\hline D6709-E & 100 & 8 & 1 & 8 & 7 & 5 & 30 & 5 \\
\hline D6709-D & 100 & $6(12)$ & - & $2(13)$ & 3 & - & $11(20)$ & (1) \\
\hline D6709-C & 200 & 15 & 3 & 3 & - & 8 & 20 & + \\
\hline D6709-B & 100 & 2 & 2 & 13 & - & 4 & 21 & - \\
\hline D6709-A & 200 & $+(4)$ & $+(6)$ & $1(10)$ & 1 & $+(4)$ & $18(45)$ & $+(12)$ \\
\hline
\end{tabular}


TABLE 2

\begin{tabular}{|c|c|c|c|c|c|c|c|c|}
\hline & 1. & 2 . & 3. & 4 & 5. & 6. & 7. & 8. \\
\hline D6710-A & 200 & 4 & 2 & 5 & 2 & 1 & 16 & - \\
\hline D6 $711-F$ & 200 & $8(8)$ & (1) & $2(3)$ & - & $+(1)$ & $5(15)$ & (2) \\
\hline D6711-E & 100 & 21 & 1 & 9 & - & + & 11 & - \\
\hline D6711-D & 200 & $3(11)$ & (5) & $2(10)$ & - & $2(5)$ & $9(38)$ & $+(2)$ \\
\hline D6711-C & 100 & 7 & - & - & - & + & 10 & + \\
\hline D6711-B & 200 & $2(4)$ & $+(1)$ & $+(2)$ & + & $2(1)$ & $2(16)$ & $+(2)$ \\
\hline D 6768 & 354 & 33 & 1 & 7 & - & 3 & 33 & + \\
\hline D6711-A & 100 & 2 & 1 & 6 & - & 1 & 12 & + \\
\hline D 6767 & 200 & 10 & 2 & 4 & - & 4 & 41 & 3 \\
\hline D6766-A & 424 & 1 & - & 2 & + & 2 & 21 & + \\
\hline D 6765 & 10 & 3 & - & - & - & + & 5 & - \\
\hline D6 764 & 200 & 7 & - & 5 & - & 4 & 14 & + \\
\hline D6763 & 7 & 7 & - & 5 & - & 3 & 35 & 3 \\
\hline D6 762 & 296 & 9 & - & 8 & - & 6 & 9 & 1 \\
\hline
\end{tabular}

continued on p. 16 
TABLE 2

10. 11. 12. 13. 14. 15. 16. 17.

\begin{tabular}{|c|c|c|c|c|c|c|c|c|}
\hline $\mathrm{D} 6707-\mathrm{CC}$ & - & - & $+(1)$ & $+(1)$ & - & - & $+(1)$ & $3(10)$ \\
\hline D $6707-B B$ & - & - & 66 & - & - & - & - & 37 \\
\hline $\mathrm{D} 6707-\mathrm{AA}$ & + & - & 3 & + & - & - & - & 6 \\
\hline D6707-z & - & - & $1(6)$ & $+(2)$ & - & - & - & (19) \\
\hline D $6707-U$ & - & - & $2(1)$ & $1(1)$ & (1) & - & $(+)$ & $2(3)$ \\
\hline D6707-T & - & - & $2(4)$ & $+(2)$ & - & $(+)$ & (1) & 14 \\
\hline D6707-Q & - & - & $+(2)$ & $1(8)$ & - & - & - & 18 \\
\hline D6707-0 & - & - & $4(3)$ & + & (1) & - & - & (1) \\
\hline D $6707-N$ & - & - & $1(1)$ & $+(2)$ & - & - & (1) & $1(20)$ \\
\hline D6707-M & + & - & (4) & $+(3)$ & - & - & (1) & $6(15)$ \\
\hline D6707-K & - & - & - & (7) & - & - & $+(17)$ & $+(16)$ \\
\hline D6707-I & - & - & $1(2)$ & 1 & - & - & + & $10(28)$ \\
\hline D $6707-E$ & - & - & 5 & + & - & - & + & + \\
\hline D6707-A & - & - & $1(4)$ & + & (1) & - & $+(2)$ & $4(10)$ \\
\hline
\end{tabular}


TABLE 2

$$
\text { 10. 11. 12. 13. 14. 15. 16. } 17 \text {. }
$$

\begin{tabular}{|c|c|c|c|c|c|c|c|c|}
\hline D6708-D & - & - & $4(4)$ & $3(2)$ & - & + & $4(7)$ & (6) \\
\hline D6 $708-B$ & - & - & $19(55)$ & - & - & - & + & + \\
\hline D $6708-A$ & 1 & - & 63 & + & - & - & - & + \\
\hline D6709-K & - & - & $1(4)$ & + & - & - & (2) & $+(1)$ \\
\hline D6709-J & - & - & + & - & - & - & - & - \\
\hline D6709-I & - & - & + & - & - & - & - & - \\
\hline D $6709-\mathrm{H}$ & - & - & 7 & 1 & - & + & 7 & 14 \\
\hline D6709-G & - & - & 23 & + & - & - & 1 & 1 \\
\hline D6709-F & - & - & 3 & - & - & - & + & 4 \\
\hline D6709-E & - & - & 10 & - & - & + & - & 1 \\
\hline D6709-D & - & - & $2(4)$ & - & - & - & $+(3)$ & (2) \\
\hline D6709-C & 1 & - & 27 & + & - & - & + & + \\
\hline D $6709-B$ & - & - & 1 & + & - & - & + & 22 \\
\hline D6709-A & + & - & $4(14)$ & 1 & + & - & - & $2(8)$ \\
\hline
\end{tabular}


TABLE 2

10. 11. 12. 13. 14. 15. 16.

\begin{tabular}{|c|c|c|c|c|c|c|c|c|}
\hline D6710-A & - & - & + & - & - & - & 1 & 19 \\
\hline D6711-F & - & - & + & + & - & - & 1 & $9(39)$ \\
\hline D6711-E & - & - & 6 & + & - & - & + & 10 \\
\hline D $6711-D$ & - & - & $4(5)$ & $+(3)$ & + & $(+)$ & + & $28(65)$ \\
\hline D $6711-C$ & - & - & 12 & - & 1 & - & - & 7 \\
\hline D6711-B & - & - & $+(1)$ & + & + & - & - & $25(61)$ \\
\hline D 6768 & - & + & 3 & 1 & 1 & - & 2 & 34 \\
\hline D6711-A & - & - & 17 & + & - & - & - & 1 \\
\hline D 6767 & + & - & 20 & 2 & + & - & + & 6 \\
\hline D6766-A & - & - & 18 & + & + & - & 1 & + \\
\hline D 6765 & - & + & 100 & 2 & - & - & - & 2 \\
\hline D 6764 & - & + & 57 & - & - & - & - & 2 \\
\hline D 6763 & - & 3 & 42 & - & - & - & - & 15 \\
\hline D 6762 & - & 4 & 18 & 2 & + & - & - & 32 \\
\hline
\end{tabular}


TAB LE 2

18. 19. 20. 21. 22. 23. 24. 25. 26.

\begin{tabular}{|c|c|c|c|c|c|c|c|c|c|}
\hline D $6707-C C$ & + & $1(11)$ & + & $(7)$ & $+(2)$ & - & $1(4)$ & - & - \\
\hline D6 707-B B & - & 2 & - & - & - & - & 23 & - & 1 \\
\hline D 6707-AA & - & 2 & - & - & - & - & 3 & - & + \\
\hline D6707-Z & $(6)$ & $9(12)$ & - & $(10)$ & (2) & - & $+(1)$ & - & - \\
\hline D6707-U & - & - & - & - & $1(3)$ & - & (2) & - & - \\
\hline D6707-T & - & + & - & - & $+(2)$ & - & $+(2)$ & - & $+(2)$ \\
\hline D6707-Q & + & $+(2)$ & (2) & $(28)$ & $+(3)$ & - & - & - & - \\
\hline D6707-0 & - & - & - & - & (2) & - & - & - & $+(1)$ \\
\hline D6707-N & + & $+(6)$ & - & + & $+(6)$ & - & + & - & + \\
\hline D6707-M & $+(2)$ & $8(20)$ & - & $(2)$ & (3) & - & $+(4)$ & - & - \\
\hline D6707-K & - & (3) & (1) & - & (2) & - & $15(34)$ & - & $(+)$ \\
\hline D6707-I & $+(1)$ & $4(12)$ & (8) & - & (3) & - & $2(2)$ & - & - \\
\hline D6707-E & - & + & - & - & - & - & - & + & 4 \\
\hline D6707-A & - & + & - & - & (4) & (1) & - & + & - \\
\hline
\end{tabular}


TABLE 2

18. 19. 20. 21. 22. 23. 24. 25. 26.

\begin{tabular}{|c|c|c|c|c|c|c|c|c|c|}
\hline D6708-D & + & $2(2)$ & - & - & - & + & 1 & - & + \\
\hline D6708-B & - & + & - & - & (1) & - & - & + & $+(2)$ \\
\hline D6708-A & - & - & - & - & - & - & 7 & + & + \\
\hline D6709-K & + & 1 & - & (1) & (4) & - & + & - & + \\
\hline D6709-J & - & - & - & - & - & - & - & - & - \\
\hline D6709-I & - & - & - & - & - & - & - & - & - \\
\hline D6709-H & 21 & 36 & 4 & 6 & - & + & 9 & - & - \\
\hline D6709-G & 1 & 1 & - & - & - & - & - & + & + \\
\hline D6709-F & + & + & - & - & 2 & - & - & + & - \\
\hline D6709-E & 8 & + & - & - & - & - & + & 1 & - \\
\hline D6709-D & (1) & (1) & - & - & - & - & - & - & - \\
\hline D6709-C & - & - & - & - & + & - & - & - & 9 \\
\hline D6709-B & - & + & - & + & - & - & - & - & - \\
\hline D6709-A & - & + & - & - & $2(4)$ & (1) & - & + & + \\
\hline
\end{tabular}


TABLE 2

18. 19. 20. 21. 22. 23. 24. 25. 26 .

\begin{tabular}{|c|c|c|c|c|c|c|c|c|c|}
\hline D6710-A & 3 & 1 & - & - & - & - & 35 & - & 27 \\
\hline D6711-F & - & + & - & (3) & $+(1)$ & (2) & - & - & - \\
\hline D $6711-E$ & - & 2 & - & + & - & - & - & - & - \\
\hline D6711-D & 2 & $4(11)$ & - & $1(3)$ & + & + & (2) & - & + \\
\hline D6711-C & 4 & 2 & - & 2 & 1 & - & - & - & + \\
\hline D6711-B & $1(2)$ & (5) & - & - & - & - & - & - & - \\
\hline D 6768 & 2 & 3 & 1 & + & + & + & 2 & - & + \\
\hline D6711-A & 2 & + & - & - & 1 & - & 2 & - & 1 \\
\hline D 6767 & - & + & - & - & - & + & + & - & + \\
\hline D6766-A & - & - & - & - & 2 & - & - & - & - \\
\hline D 6765 & - & - & - & - & - & - & - & - & - \\
\hline D 6764 & - & - & - & - & - & - & - & - & - \\
\hline D 6763 & - & - & - & - & - & - & + & - & - \\
\hline D6 762 & - & - & - & - & - & + & - & - & - \\
\hline
\end{tabular}


TAB LE 2

27. 28. 29. 30. 31. 32. 33. 34. 35.

\begin{tabular}{|c|c|c|c|c|c|c|c|c|}
\hline D6707-CC & $1(1)$ & - & 3 & (6) & - & + & - & $1(3)$ \\
\hline D6 707-B B & - & - & - & 40 & 1 & - & - & 5 \\
\hline D6707-AA & - & 18 & - & 32 & - & 11 & - & 2 \\
\hline D6707-Z & $1(1)$ & - & (8) & $14(3)$ & - & (1) & - & (5) \\
\hline D6707-U & - & $26(50)$ & $(10)$ & $18(14)$ & $+(1)$ & $8(13)$ & + & $3(3)$ \\
\hline D6707-T & + & $+(1)$ & $7(5)$ & $7(18)$ & - & 1 & (2) & (4) \\
\hline D6707-Q & $+(3)$ & - & 2 & $4(9)$ & - & + & - & (3) \\
\hline D6707-0 & - & $1(1)$ & 4 & $13(28)$ & - & - & - & 1 \\
\hline D6707-N & + & - & $10(6)$ & $2(19)$ & - & - & (1) & $4(16)$ \\
\hline D6707-M & $+(6)$ & $(1)$ & $12(13)$ & $(11)$ & 2 & - & - & $2(9)$ \\
\hline D6707-K & - & (2) & $6(4)$ & $5(12)$ & (1) & - & - & $+(4)$ \\
\hline D6707-I & $+(1)$ & - & $4(16)$ & $4(12)$ & - & - & - & $3(12)$ \\
\hline D6707-E & - & - & 9 & 4 & 4 & + & - & 3 \\
\hline D6707-A & - & (2) & $4(6)$ & $7(37)$ & 1 & - & - & $3(17)$ \\
\hline
\end{tabular}




$$
\text { 27. 28. 29. 30. 31. 32. 33. 34. } 35 \text {. }
$$

\begin{tabular}{|c|c|c|c|c|c|c|c|c|c|}
\hline D6708-D & - & $2(2)$ & 4 & $5(13)$ & + & $+(5)$ & - & $2(10)$ & - \\
\hline D6 $708-B$ & - & $+(1)$ & + & $14(37)$ & + & $+(1)$ & - & $3(5)$ & - \\
\hline D6708-A & - & 6 & 5 & 21 & - & 2 & - & 12 & - \\
\hline D6709-K & + & $4(21)$ & + & $8(18)$ & (5) & + & - & $+(10)$ & - \\
\hline D6709-J & - & - & + & + & - & - & - & - & - \\
\hline D6709-I & - & - & - & - & - & - & - & - & - \\
\hline D6709-H & + & - & 61 & 14 & 7 & 3 & - & 9 & + \\
\hline D6709-G & - & - & 89 & + & 7 & 1 & + & 10 & - \\
\hline D6709-F & - & 2 & 56 & 6 & 3 & + & - & 3 & - \\
\hline D6709-E & - & + & 65 & 2 & 4 & 3 & - & 6 & - \\
\hline D6709-D & $+(4)$ & (3) & $2(12)$ & $5(17)$ & - & - & - & $2(5)$ & $2(1)$ \\
\hline D6709-C & - & 5 & 11 & 13 & 2 & 1 & + & 5 & - \\
\hline D6709-B & 1 & - & 10 & - & - & - & - & 11 & 1 \\
\hline D6709-A & + & $7(21)$ & - & $14(38)$ & - & (5) & $1(6)$ & $3(5)$ & + \\
\hline
\end{tabular}


TABLE 2

\begin{tabular}{|c|c|c|c|c|c|c|c|c|c|}
\hline & 27 & 28 & 29. & 30. & 31. & 32. & 33. & 34. & 35 \\
\hline D $6710-A$ & - & - & 10 & + & 6 & - & - & 1 & - \\
\hline D6711-F & + & - & 1 & $1(3)$ & (2) & $1(5)$ & - & $2(8)$ & - \\
\hline D6711-E & 2 & - & 28 & 1 & - & - & - & + & - \\
\hline D6711-D & - & - & $9(31)$ & $3(36)$ & + & - & 1 & $1(3)$ & - \\
\hline D6711-C & + & - & 35 & 36 & - & - & - & + & - \\
\hline D6711-B & $+(1)$ & 2 & $+(2)$ & - & + & - & - & (1) & - \\
\hline D6768 & + & + & - & 6 & 2 & + & - & - & + \\
\hline D67611-A & - & 6 & + & 119 & - & 55 & - & 2 & - \\
\hline D 6767 & - & 5 & + & 48 & 2 & 1 & 1 & + & - \\
\hline D6766-A & - & 1 & 10 & 35 & - & 1 & - & + & - \\
\hline D 6765 & - & - & - & - & - & - & - & - & - \\
\hline D6 764 & - & - & - & 5 & - & - & - & - & - \\
\hline D6763 & - & - & - & 4 & 2 & - & - & 26 & - \\
\hline D 6762 & - & - & - & 5 & 10 & - & - & - & + \\
\hline
\end{tabular}


TABLE 2

36 .

37.38

39.

40.

41.

42.

43.

44.

\begin{tabular}{|c|c|c|c|c|c|c|c|c|c|}
\hline D $6707-C C$ & - & - & (1) & + & - & - & - & - & - \\
\hline D6707-B B & - & - & 2 & - & 3 & 2 & + & - & 3 \\
\hline D $6707-\mathrm{AA}$ & + & - & 3 & 3 & + & - & 2 & - & 1 \\
\hline D6707-Z & - & - & - & + & - & - & + & - & 1 \\
\hline D $6707-U$ & + & - & + & + & + & - & 1 & + & + \\
\hline D6 $707-T$ & - & - & - & + & - & - & + & (1) & - \\
\hline D6707-Q & - & - & - & (1) & - & - & - & - & (3) \\
\hline D6707-0 & + & - & - & 1 & - & - & - & - & 1 \\
\hline D $6707-N$ & + & - & + & $+(2)$ & + & - & + & - & + \\
\hline D6707-M & - & - & - & - & (1) & - & + & - & - \\
\hline D $6707-K$ & $+(1)$ & 2 & - & - & - & - & + & - & - \\
\hline D6707-I & - & + & + & - & - & - & - & - & - \\
\hline D6707-E & + & - & + & + & 1 & + & - & - & + \\
\hline D6707-A & - & - & - & $+(4)$ & - & + & + & - & - \\
\hline
\end{tabular}


TABLE 2

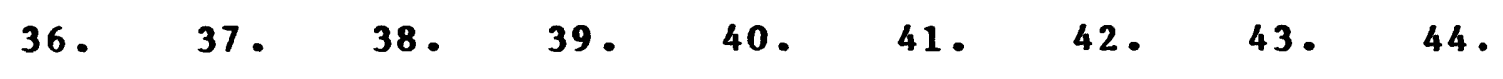

\begin{tabular}{|c|c|c|c|c|c|c|c|c|c|}
\hline D6708-D & + & - & - & 1 & $1(1)$ & + & + & - & $+(3)$ \\
\hline D6708-B & + & - & - & - & 2 & - & $+(1)$ & - & + \\
\hline D6708-A & + & - & + & 2 & 1 & - & + & - & + \\
\hline D6709-K & - & - & - & + & - & $+(1)$ & - & - & + \\
\hline D6709-J & - & - & - & - & - & + & - & - & - \\
\hline D6709-I & - & - & - & - & - & - & - & - & - \\
\hline D $6709-H$ & - & + & 1 & 2 & - & + & - & + & 1 \\
\hline D6709-G & - & - & - & - & 1 & - & - & - & - \\
\hline D6709-F & + & + & - & - & - & - & + & - & - \\
\hline D6709-E & - & - & - & - & - & 1 & - & - & - \\
\hline D6709-D & - & - & - & $1(1)$ & - & - & - & - & (2) \\
\hline D6709-C & + & - & - & - & - & + & + & - & - \\
\hline D $6709-B$ & - & - & 4 & + & - & - & - & - & - \\
\hline D6709-A & (1) & + & - & + & + & + & + & + & - \\
\hline
\end{tabular}


TABLE 2

36. 37. 38. 39. 40. 41. 42. 43. 44 .

\begin{tabular}{|c|c|c|c|c|c|c|c|c|}
\hline D6710-A & + & - & - & - & - & - & - & - \\
\hline D6 $711-F$ & - & - & 1 & - & - & - & - & - \\
\hline D6711-E & + & - & - & - & - & - & - & - \\
\hline D6711-D & (2) & - & - & - & - & - & + & - \\
\hline D6711-C & - & - & + & - & - & - & - & - \\
\hline D6711-B & - & - & - & - & - & - & + & - \\
\hline D 6768 & - & - & + & - & - & - & + & - \\
\hline D6711-A & - & - & - & 1 & - & - & - & - \\
\hline D 6767 & - & - & 1 & - & - & - & + & - \\
\hline D6766-A & - & - & - & - & 1 & - & - & - \\
\hline D 6765 & - & - & - & - & - & - & - & - \\
\hline D6 764 & - & - & - & - & - & - & - & - \\
\hline D 6763 & - & - & - & - & - & - & - & - \\
\hline D6 762 & - & - & - & - & - & - & - & - \\
\hline
\end{tabular}


TABLE 2

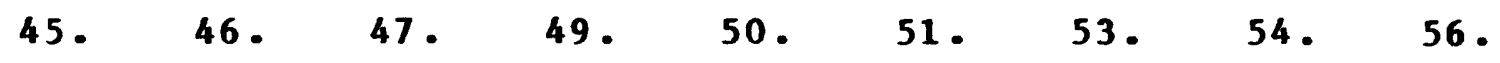

$D 6707-C C$

D $6707-B B$

D6707-AA

$+$

D6707-Z

D6707-U

$-\quad-\quad+(7)$

D6707-T

$-$

-

(2)

(1)

D6707-Q

D6707-0

D6707-N

D6707-M

D $6707-K$

D6707-I

D6707-E

D6707-A 


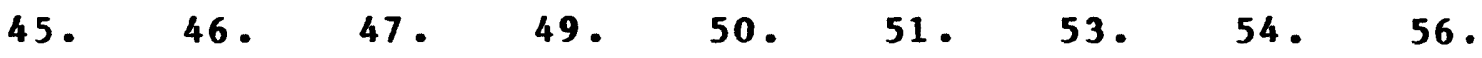

\begin{tabular}{|c|c|c|c|c|c|c|c|c|c|}
\hline D6708-D & - & - & - & - & - & - & - & - & - \\
\hline D6708-B & - & - & + & - & - & - & - & 1 & - \\
\hline D $6708-A$ & - & - & + & - & - & - & - & - & - \\
\hline D6709-K & - & - & $+(1)$ & - & - & - & - & (1) & - \\
\hline D6709-J & - & - & - & - & - & - & - & - & - \\
\hline D6709-I & - & - & - & - & - & - & - & - & - \\
\hline D $6709-\mathrm{H}$ & - & + & - & - & + & - & - & - & - \\
\hline D6709-G & - & - & - & - & - & - & - & - & - \\
\hline D6709-F & - & - & + & - & - & - & - & - & - \\
\hline D6709-E & - & - & - & - & + & - & - & - & - \\
\hline D6709-D & - & - & - & - & - & - & - & - & - \\
\hline D6709-C & - & - & - & - & - & - & - & - & - \\
\hline D6709-B & - & + & - & - & - & - & - & 1 & - \\
\hline D6709-A & (4) & - & - & - & - & - & - & - & - \\
\hline
\end{tabular}




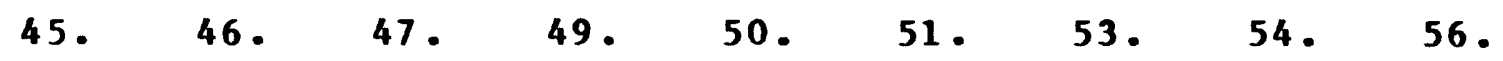

D $6710-A$

D6711-F

D6711-E

D6711-D

D6711-C

D6711-B

D6768

D6711-A

D6767

D6 766-A

D6765

D6764

D6763

D6762 
TABLE 2

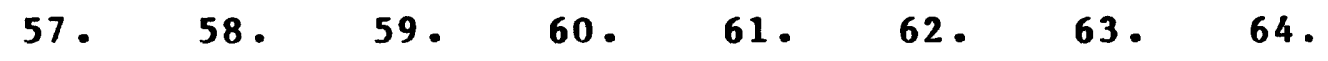

\begin{tabular}{|c|c|c|c|c|c|c|c|c|}
\hline D6707-CC & $(7)$ & - & - & $+(6)$ & (1) & 3 & - & - \\
\hline D6 $707-B \mathrm{~B}$ & 2 & 9 & - & - & - & 6 & - & - \\
\hline D6707-AA & 6 & 7 & 2 & 5 & 1 & 2 & - & (1) \\
\hline D6707-Z & - & - & - & - & $+(1)$ & $+(1)$ & - & + \\
\hline D $6707-U$ & - & (1) & - & $1(1)$ & - & $+(1)$ & - & $+(2)$ \\
\hline D6707-T & $+(27)$ & + & - & + & - & (1) & - & $+(1)$ \\
\hline D6707-Q & $2(3)$ & 3 & - & $1(1)$ & - & $+(9)$ & - & - \\
\hline D $6707-0$ & - & - & - & - & - & $+(2)$ & - & (1) \\
\hline D $6707-N$ & 1 & $+(2)$ & 1 & $+(8)$ & $2(1)$ & + & - & - \\
\hline D6707-M & (3) & - & - & - & $1(2)$ & - & + & - \\
\hline D $6707-K$ & 3 & $2(3)$ & $(+)$ & + & $+(1)$ & 1 & - & - \\
\hline D6707-I & (3) & (1) & + & - & $4(2)$ & + & + & + \\
\hline D6707-E & 1 & + & + & + & + & + & - & + \\
\hline D $6707-A$ & $4(1)$ & (1) & $+(2)$ & + & + & $1(1)$ & - & - \\
\hline
\end{tabular}


TABLE 2

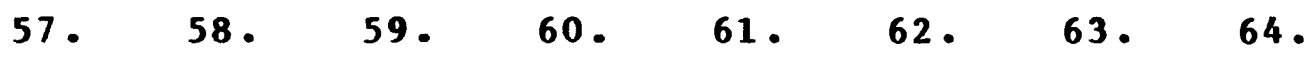

\begin{tabular}{|c|c|c|c|c|c|c|c|c|}
\hline D6708-D & - & + & $+(3)$ & - & + & + & - & - \\
\hline D6708-B & - & (1) & $1(2)$ & + & + & $+(3)$ & - & - \\
\hline D $6708-A$ & - & 8 & 1 & 1 & 1 & + & - & - \\
\hline D6709-K & (5) & $1(2)$ & $+(4)$ & $+(5)$ & + & - & - & - \\
\hline D6709-J & - & + & - & - & - & - & - & - \\
\hline D6709-I & - & + & - & - & - & - & - & - \\
\hline D $6709-\mathrm{H}$ & - & + & - & - & + & - & - & - \\
\hline D6709-G & 1 & 1 & - & - & - & 1 & - & - \\
\hline D $6709-F$ & - & + & - & + & - & - & - & - \\
\hline D6709-E & - & - & - & - & - & + & - & - \\
\hline D $6709-D$ & - & 2 & - & - & - & $2(8)$ & - & - \\
\hline D6709-C & - & 2 & + & + & - & + & - & - \\
\hline D6709-B & - & - & - & + & 1 & 1 & - & - \\
\hline D6709-A & $6(4)$ & $+(4)$ & - & (1) & - & $2(1)$ & - & - \\
\hline
\end{tabular}


TABLE 2

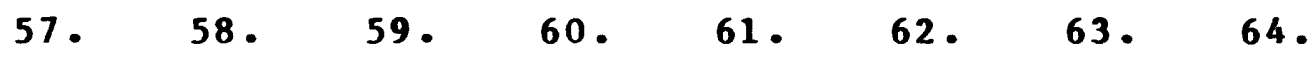

\begin{tabular}{|c|c|c|c|c|c|c|c|c|}
\hline D6710-A & - & + & - & - & - & 5 & - & - \\
\hline D6711-F & $2(6)$ & (1) & - & - & - & - & - & - \\
\hline D6711-E & 8 & - & - & - & - & - & - & + \\
\hline D6711-D & (3) & - & - & $+(2)$ & - & $+(7)$ & - & - \\
\hline D6711-C & 1 & - & - & - & - & 10 & - & - \\
\hline D6711-B & $2(10)$ & - & + & - & 1 & (2) & - & + \\
\hline D 6768 & 4 & + & - & 2 & + & 6 & - & - \\
\hline D6711-A & - & - & - & - & - & 3 & + & - \\
\hline D 6767 & 9 & 5 & - & + & - & 5 & - & - \\
\hline D6766-A & 4 & 1 & - & + & - & 1 & - & - \\
\hline D6765 & 4 & - & - & - & - & 23 & - & - \\
\hline D 6764 & 2 & - & - & - & - & 26 & - & - \\
\hline D 6763 & 12 & - & - & - & - & 35 & - & - \\
\hline D 6762 & - & - & - & - & - & - & - & - \\
\hline
\end{tabular}


TABLE 3

PALYNOMORPHS IN THE ANDERSON COAL ZONE

\section{USGS Paleobotany}

locality no.

Palynomorph number

1. 2. 3. 4. 5. 6. $\quad$ 4. 8. 10. $12 \cdot 13$.

\begin{tabular}{|c|c|c|c|c|c|c|c|c|c|c|c|}
\hline D6707-N' & 434 & 1 & 2 & 3 & 2 & - & + & - & - & + & - \\
\hline D6707-M' & 234 & 9 & 16 & 9 & 1 & - & 3 & + & - & 1 & - \\
\hline D6707-L & 338 & 15 & 2 & 2 & 1 & - & 8 & + & - & - & 2 \\
\hline D6707-J & 305 & 7 & 12 & 6 & 1 & - & 22 & + & - & 1 & + \\
\hline D6707-H & 329 & 18 & 9 & 15 & - & - & 7 & + & - & 1 & 1 \\
\hline D6707-G & 347 & + & 12 & 8 & + & + & 6 & 2 & - & 2 & + \\
\hline D $6707-F$ & 394 & 19 & 8 & 5 & 1 & + & 6 & 2 & - & + & - \\
\hline D6707-D & 362 & 7 & 10 & 5 & 3 & + & 6 & 1 & + & + & 3 \\
\hline D $6707-C$ & 240 & 11 & 8 & 10 & - & - & 20 & + & - & 4 & 1 \\
\hline D6707-B & 312 & 3 & 9 & 12 & - & 2 & 16 & + & - & 4 & 1 \\
\hline
\end{tabular}

D6710-E

$\begin{array}{lllllllll}218 & 22 & 3 & 3 & 2 & - & 8 & 1 & -\end{array}$

D6710-D

38416

101

12

$-$

$-13$

1

$-\quad+$

2

D6710-C

350

$21 \quad 12 \quad 9$

D6710-B

372

$8 \quad 7$

2

2

3

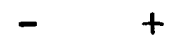

8

continued on p. 35 
TABLE 3

1. 2. 3. 4. 5. 6. $\quad$ 4. $\quad$ 8. 10. 12. 13.

\begin{tabular}{|c|c|c|c|c|c|c|c|c|c|c|c|}
\hline D6708-0 & 300 & 9 & 9 & 2 & - & 2 & 1 & - & - & 3 & 2 \\
\hline D6 $708-N$ & 354 & 11 & 4 & - & - & - & 10 & 1 & - & - & + \\
\hline D6708-M & 260 & 16 & 14 & 8 & - & + & 17 & + & - & 2 & + \\
\hline D6708-L & 319 & 1 & 9 & 3 & - & + & 7 & - & - & + & + \\
\hline D6708-K & 327 & 42 & 16 & 6 & - & - & 18 & + & - & 2 & 1 \\
\hline D6 708-J & 368 & 18 & 11 & 7 & - & 1 & 5 & 1 & - & 2 & + \\
\hline D6708-I & 244 & 6 & 30 & 15 & 3 & - & 19 & 2 & - & 3 & + \\
\hline D6 $708-H$ & 303 & 29 & 11 & 14 & - & + & 22 & - & - & + & + \\
\hline D6708-G & 179 & 17 & 37 & 27 & - & 3 & 14 & 3 & - & 9 & - \\
\hline D $6708-F$ & 284 & 2 & 7 & 17 & - & 2 & 13 & 3 & - & 3 & + \\
\hline D6708-E & 350 & 1 & 11 & 5 & - & 2 & 16 & 2 & - & 1 & 2 \\
\hline D6708-C & 383 & 6 & 10 & 15 & - & + & 8 & 3 & - & 4 & 1 \\
\hline
\end{tabular}


TABLE 3

14. 15. 16. 17. 18. 19. 20. 21. 22. 23. 24 .

\begin{tabular}{|c|c|c|c|c|c|c|c|c|c|c|c|}
\hline D $6707-N^{\prime}$ & - & - & - & 18 & 8 & 2 & 5 & - & 1 & - & - \\
\hline D6707-M' & - & + & 4 & 12 & 13 & 51 & 1 & 5 & - & - & 4 \\
\hline D6707-L & - & - & 2 & 5 & - & 19 & 1 & 5 & 2 & 1 & 34 \\
\hline D6707-J & 2 & + & 1 & 33 & + & 41 & + & + & + & 1 & + \\
\hline D6 $707-\mathrm{H}$ & - & + & + & 30 & 1 & 35 & 1 & 9 & 2 & - & + \\
\hline D6707-G & 1 & - & - & 15 & - & 34 & + & 3 & + & - & + \\
\hline D6707-F & + & - & - & 10 & - & 4 & 2 & - & 5 & 1 & 1 \\
\hline D6 707-D & - & - & + & 8 & - & 13 & 1 & - & - & - & 2 \\
\hline D6707-C & + & 1 & 2 & 5 & 11 & 21 & 2 & - & - & - & + \\
\hline D6707-B & + & - & 1 & 3 & 6 & 2 & - & - & - & + & + \\
\hline D6710-E & + & - & + & 8 & 2 & 38 & 3 & 29 & 2 & + & 90 \\
\hline D6710-D & 1 & - & + & 12 & 2 & 13 & 1 & 4 & 2 & 1 & + \\
\hline D6710-C & + & - & 1 & 26 & + & 26 & 1 & 3 & 2 & + & - \\
\hline D6710-B & + & + & 1 & 13 & + & 16 & - & - & - & - & - \\
\hline
\end{tabular}


TABLE 3

14. 15. 16. 17. 18. 19. 20. 21. 22. 23. 24.

\begin{tabular}{|c|c|c|c|c|c|c|c|c|c|c|c|}
\hline D6708-0 & - & - & + & 2 & 8 & 55 & 3 & 15 & - & + & 3 \\
\hline D6708-N & 1 & - & - & 2 & - & 13 & - & 14 & - & + & 46 \\
\hline D6708-M & + & + & + & 10 & 2 & 40 & 5 & + & + & + & 8 \\
\hline D6708-L & 1 & - & + & 4 & 1 & 45 & 4 & 3 & 1 & - & - \\
\hline D6708-K & - & - & 1 & 26 & 1 & 34 & 3 & + & 1 & 1 & - \\
\hline D6708-J & - & - & 1 & 24 & - & 23 & 2 & 3 & 3 & + & + \\
\hline D6708-I & 1 & + & + & 16 & 6 & 57 & 11 & 16 & 2 & - & 2 \\
\hline D6 $708-H$ & - & + & + & 32 & + & 22 & 4 & 9 & 1 & - & 5 \\
\hline D6708-G & 2 & - & 3 & 7 & + & 32 & 2 & - & - & 1 & 95 \\
\hline D6708-F & + & - & + & 5 & 8 & 20 & 2 & - & - & - & - \\
\hline D6708-E & - & 1 & + & 3 & 1 & 13 & - & - & 2 & + & + \\
\hline D6708-C & + & - & 1 & 2 & - & 7 & - & - & 9 & - & - \\
\hline
\end{tabular}


25. 26. 27. 28. 29. 30. 31. 32. 33. 34. 35. 36.

\begin{tabular}{|c|c|c|c|c|c|c|c|c|c|c|c|c|}
\hline D $6707-N^{\prime}$ & - & - & - & - & - & 3 & 5 & - & - & 1 & 1 & - \\
\hline D6707-M' & - & - & 3 & + & 111 & + & 4 & - & - & 4 & + & + \\
\hline D6707-L & + & + & 1 & 28 & - & 8 & - & 4 & - & 2 & - & + \\
\hline D6707-J & - & + & 4 & + & 25 & 2 & 2 & - & - & 7 & 1 & - \\
\hline D6 707-H & - & + & 4 & - & 7 & 2 & 4 & - & - & 9 & + & - \\
\hline D6707-G & - & - & 8 & - & 28 & 3 & 13 & - & - & 6 & - & - \\
\hline D $6707-F$ & - & - & 1 & 1 & 4 & 7 & 9 & - & - & + & - & + \\
\hline D6707-D & - & + & 1 & - & 46 & 9 & 3 & - & + & 1 & - & - \\
\hline D $6707-C$ & - & + & + & 4 & 143 & 22 & 1 & - & - & 4 & - & - \\
\hline D6707-B & - & - & - & 45 & - & 37 & 9 & - & - & 8 & - & - \\
\hline D6710-E & - & - & 5 & 4 & 24 & 6 & - & - & - & - & - & - \\
\hline D6710-D & - & + & + & 1 & 3 & 7 & + & - & - & 5 & - & - \\
\hline D6710-C & - & - & 4 & - & 10 & 5 & 7 & - & - & 12 & - & - \\
\hline D6710-B & - & - & 1 & 3 & 48 & 4 & 2 & - & - & 3 & - & - \\
\hline
\end{tabular}


TABLE 3

25. 26. 27. 28. 29. 30. 31. 32. 33. 34. 35. 36.

\begin{tabular}{|c|c|c|c|c|c|c|c|c|c|c|c|c|}
\hline D $6708-0$ & - & - & 4 & - & 44 & 4 & 12 & - & - & 10 & - & - \\
\hline D6708-N & - & + & 2 & + & 2 & 18 & 5 & - & - & 2 & - & - \\
\hline D $6708-M$ & - & - & + & + & 68 & 16 & 10 & - & - & 12 & 2 & + \\
\hline D6 $708-L$ & - & - & 1 & + & 94 & 3 & - & - & - & 3 & - & + \\
\hline D6708-K & - & - & 2 & - & 6 & 5 & 2 & - & - & 1 & - & - \\
\hline D6708-J & - & - & - & - & 6 & 7 & 1 & 1 & - & 12 & - & - \\
\hline D6708-I & - & + & 10 & - & 12 & 4 & 2 & 1 & - & 17 & 1 & + \\
\hline D6 $708-H$ & - & - & 10 & - & 4 & 2 & 13 & - & - & 5 & - & - \\
\hline D6708-G & + & - & 4 & 4 & - & 20 & 13 & - & - & 6 & - & 2 \\
\hline D6708-F & - & 1 & 2 & 7 & 78 & 28 & 1 & 4 & - & 5 & + & - \\
\hline D6708-E & - & - & - & 11 & 42 & 17 & 1 & 2 & - & 10 & - & - \\
\hline D6 $708-C$ & - & + & - & 8 & 5 & 28 & 1 & - & - & 7 & - & + \\
\hline
\end{tabular}


TABLE 3

37. $38 \cdot 39.40 \cdot 41 \cdot 42.43 \cdot 45 \cdot 46 \cdot 48 \cdot 49.50$.

\begin{tabular}{|c|c|c|c|c|c|c|c|c|c|c|c|c|}
\hline D6707-N' & - & - & - & - & - & - & - & - & - & - & - & + \\
\hline D6707-M' & - & 2 & - & - & 1 & - & - & - & + & - & - & - \\
\hline D6707-L & + & 6 & - & - & - & + & + & - & + & - & - & - \\
\hline D6707-J & - & 1 & - & - & - & - & - & - & + & - & - & - \\
\hline D6707-H & - & - & - & - & - & + & - & - & - & + & - & - \\
\hline D6707-G & - & - & + & - & - & - & - & - & - & - & - & - \\
\hline D6707-F & - & 3 & - & - & - & + & - & - & 1 & - & - & - \\
\hline D6707-D & - & 3 & 1 & - & - & + & - & - & + & - & - & - \\
\hline D6707-C & - & 1 & - & + & - & - & - & - & + & - & - & 1 \\
\hline D6707-B & - & 1 & 1 & - & 2 & + & + & - & + & - & - & - \\
\hline D6710-E & - & 15 & - & - & - & + & - & - & - & - & - & - \\
\hline D6710-D & - & - & 1 & + & - & - & - & - & - & - & - & - \\
\hline D $6710-C$ & - & + & - & - & 1 & - & - & - & - & - & - & - \\
\hline D6710-B & - & + & - & - & - & - & - & - & - & 1 & - & - \\
\hline
\end{tabular}


TABLE 3

37. 38. 39. 40. 41. 42. 43. 45. 46. 48. 49. 50.

\begin{tabular}{|c|c|c|c|c|c|c|c|c|c|c|c|c|}
\hline D $6708-0$ & - & 2 & - & - & - & - & - & - & - & - & - & - \\
\hline D6708-N & - & 2 & - & + & - & 1 & + & - & - & - & - & - \\
\hline D6708-M & - & + & - & - & 1 & - & - & - & - & - & - & - \\
\hline D6708-L & + & - & 1 & - & - & - & - & - & - & - & - & - \\
\hline D $6708-K$ & - & - & - & - & - & - & - & - & - & - & - & - \\
\hline D6708-J & + & - & - & - & - & - & - & - & - & - & - & - \\
\hline D6708-I & + & - & - & - & - & - & - & - & - & - & - & - \\
\hline D6708-H & 1 & - & - & - & - & + & - & - & - & - & - & - \\
\hline D $6708-G$ & - & - & - & - & - & - & - & - & - & - & - & - \\
\hline D6708-F & - & 2 & 1 & - & - & - & + & - & - & - & 2 & - \\
\hline D6708-E & - & - & + & - & - & 2 & - & + & - & - & - & - \\
\hline D6708-C & - & 2 & - & - & - & + & + & - & + & - & - & - \\
\hline
\end{tabular}


54. 57. 58. 59.60.61.62.63.64.

\begin{tabular}{|c|c|c|c|c|c|c|c|c|}
\hline D6707-N' & - & 10 & 1 & - & - & 1 & 1 & - \\
\hline D6707-M' & - & 7 & 1 & + & - & 2 & - & - \\
\hline D $6707-\mathrm{L}$ & - & 6 & 5 & + & + & 3 & - & - \\
\hline D6707-J & - & 14 & 3 & 1 & + & 5 & 3 & - \\
\hline D $6707-\mathrm{H}$ & - & 9 & + & 2 & + & 1 & 1 & - \\
\hline D6707-G & - & 8 & - & - & 1 & 1 & - & - \\
\hline D6707-F & - & - & 4 & 4 & 2 & 3 & 4 & - \\
\hline D6707-D & - & 14 & + & + & + & - & 1 & - \\
\hline D $6707-C$ & + & 5 & + & + & - & + & 2 & - \\
\hline D6707-B & 2 & 15 & + & + & + & + & 5 & - \\
\hline D6710-E & - & 9 & + & - & - & 1 & 5 & - \\
\hline D6710-D & - & 2 & 2 & + & 4 & + & 1 & - \\
\hline D $6710-C$ & - & 1 & 1 & - & 1 & + & + & + \\
\hline D6710-B & - & 4 & 4 & - & + & 1 & 1 & - \\
\hline
\end{tabular}


TABLE 3

54. 57. 58. 59.60.61.62.63.64.

\begin{tabular}{|c|c|c|c|c|c|c|c|c|}
\hline D $6708-0$ & - & - & - & - & - & 10 & - & - \\
\hline D6708-N & - & 4 & + & + & - & 3 & 3 & - \\
\hline D6708-M & - & - & 3 & 3 & 1 & 2 & + & - \\
\hline D6 $708-L$ & - & - & - & - & + & + & + & - \\
\hline D6708-K & - & - & + & - & 1 & + & 4 & - \\
\hline D6708-J & - & 2 & + & - & - & 1 & - & - \\
\hline D6708-I & - & 8 & 1 & 1 & - & 4 & 5 & - \\
\hline D6 $608-\mathrm{H}$ & - & 4 & 5 & - & 1 & 2 & 1 & - \\
\hline D $6708-G$ & - & 4 & 1 & - & - & 11 & 4 & - \\
\hline D6708-F & + & - & 2 & - & - & 1 & 1 & - \\
\hline D $6708-E$ & + & 4 & 1 & - & + & - & + & + \\
\hline D6708-C & - & - & - & + & 1 & + & + & - \\
\hline
\end{tabular}


TABLE 4

PALYNOMORPHS IN THE SMITH COAL ZONE

\section{USGS Paleobotany}

locality no.

Palynomorph number

\begin{tabular}{|c|c|c|c|c|c|c|c|c|c|c|c|}
\hline & 1. & 2 & 3. & 4. & 5. & 6. & 7 . & 8 & 10 & 12. & 13 \\
\hline D6720-I & 291 & 6 & 12 & 16 & 3 & + & 14 & + & - & 3 & 1 \\
\hline D6 $720-\mathrm{H}$ & 389 & 11 & 2 & 2 & - & - & 1 & 1 & - & + & 1 \\
\hline D6720-G & 318 & 12 & 6 & 4 & 2 & - & 7 & 1 & - & 4 & + \\
\hline D6720-F & 264 & 9 & 2 & 4 & - & + & 2 & - & - & 6 & 3 \\
\hline D6 $6720-E$ & 502 & 5 & - & - & - & - & 2 & - & - & 2 & + \\
\hline D6720-D & 350 & 11 & 5 & 3 & - & - & 3 & - & - & 21 & - \\
\hline D $6720-C$ & 230 & 7 & 7 & - & 1 & 2 & 7 & + & - & 52 & 1 \\
\hline D6 $620-B$ & 339 & 13 & 1 & - & - & - & 2 & - & - & 1 & 2 \\
\hline D $6720-A$ & 266 & 8 & 1 & 2 & - & - & + & - & - & 15 & 32 \\
\hline
\end{tabular}

continued on p. 45 
TABLE 4

\begin{tabular}{|c|c|c|c|c|c|c|c|c|c|c|c|}
\hline & 1 . & 2. & 3. & 4. & 5. & 6. & 7. & 8. & 10 & 12. & 13. \\
\hline D $6707-Z^{\prime}$ & 399 & 4 & 5 & 3 & - & - & 12 & + & - & 1 & 1 \\
\hline D6707-Y & 370 & 15 & - & 4 & - & - & 14 & - & - & 1 & - \\
\hline D6707-X & 384 & 29 & 2 & 2 & - & - & 9 & + & - & 1 & 2 \\
\hline D6 $707-W$ & 321 & 10 & 2 & 6 & - & - & 6 & - & - & - & 1 \\
\hline D6 $707-V$ & 294 & 11 & 5 & 14 & + & 1 & 8 & 1 & - & 10 & 12 \\
\hline D6707-S & 246 & 31 & 6 & 3 & - & - & 7 & - & - & 5 & - \\
\hline D6707-R & 385 & 21 & 1 & 9 & - & - & 8 & - & - & + & 1 \\
\hline D6707-P & 348 & 41 & + & 4 & 1 & - & 4 & + & - & 3 & + \\
\hline
\end{tabular}


TAB LE 4

14. 15. 16. 17. 18. 19. 20. 21. 22. 23. 24. 26 .

\begin{tabular}{|c|c|c|c|c|c|c|c|c|c|c|c|}
\hline D $6720-I$ & + & + & + & 7 & 2 & 39 & 4 & 4 & + & 1 & + \\
\hline D6 720-H & - & - & - & 9 & - & 19 & + & 3 & 7 & - & + \\
\hline D $6720-G$ & + & + & 1 & 11 & - & 4 & 5 & 2 & 2 & 3 & 4 \\
\hline D6720-F & 1 & 1 & 1 & 9 & 1 & 7 & 3 & 4 & - & 1 & - \\
\hline D6720-E & 1 & + & - & 4 & - & 5 & 2 & - & 5 & - & - \\
\hline D6720-D & + & - & - & 1 & - & 2 & - & - & - & - & 6 \\
\hline D $6720-C$ & - & - & + & 3 & - & 2 & - & - & + & 1 & + \\
\hline D6720-B & + & - & - & 8 & - & 2 & 2 & 1 & 4 & - & + \\
\hline D $6720-A$ & 2 & 2 & - & 2 & - & 2 & 3 & - & + & + & 1 \\
\hline
\end{tabular}


TAB LE 4

14. 15. 16. 17. 18. 19. 20. 21. 22. 23. 24. 26.

\begin{tabular}{|c|c|c|c|c|c|c|c|c|c|c|c|}
\hline D $6707-Z^{\prime}$ & 1 & - & - & 3 & + & 22 & 1 & 1 & 6 & 1 & + \\
\hline D6707-Y & - & - & - & 6 & - & 3 & 2 & - & 7 & - & - \\
\hline D6707-X & - & - & - & 15 & 2 & 4 & - & + & 5 & - & - \\
\hline D6707-W & 3 & - & + & 11 & + & 9 & 5 & 3 & 11 & - & + \\
\hline D6707-V & 1 & 2 & + & 21 & + & 10 & 2 & - & 1 & + & 3 \\
\hline D6707-S & 1 & 1 & - & 11 & 4 & 21 & 3 & - & - & - & + \\
\hline D6707-R & - & + & + & 7 & - & 4 & 3 & 1 & 1 & 1 & - \\
\hline D6707-P & - & - & - & 4 & - & - & 1 & - & - & 1 & 2 \\
\hline
\end{tabular}


TAB LE 4

27. 28. 29. 30. 31. 32. 33. 34. $35 \cdot 37 \cdot 38 \cdot 39$.

\begin{tabular}{|c|c|c|c|c|c|c|c|c|c|c|c|}
\hline D6720-I & 2 & 1 & 38 & 9 & 4 & - & - & 19 & - & - & 2 \\
\hline D6720-H & 2 & - & 3 & + & 5 & 1 & - & 7 & - & - & + \\
\hline D $6720-G$ & 4 & - & 36 & 15 & 7 & - & - & 4 & 2 & - & 3 \\
\hline D6720-F & 1 & 2 & 114 & 36 & - & 4 & - & 1 & - & 2 & 7 \\
\hline D6720-E & - & 5 & - & 32 & 4 & 1 & - & 5 & - & - & 5 \\
\hline D6720-D & - & 6 & - & 50 & - & 3 & - & 1 & - & - & - \\
\hline D $6720-C$ & - & 12 & 4 & 107 & - & 3 & - & - & - & - & 6 \\
\hline D6720-B & 2 & - & 1 & 19 & - & 1 & - & 1 & - & - & 1 \\
\hline D 6720-A & + & 28 & 39 & 48 & - & 15 & - & - & + & - & 3 \\
\hline
\end{tabular}


TAB LE 4

27. 28. 29. 30. 31. 32. 33. 34. $35 \cdot 37 \cdot 38 \cdot 39 \cdot$

\begin{tabular}{|c|c|c|c|c|c|c|c|c|c|c|c|}
\hline D6707-Z' & 2 & - & 9 & 15 & 4 & - & - & 6 & - & - & - \\
\hline D6707-Y & 1 & - & 3 & 9 & 18 & - & - & - & 1 & - & - \\
\hline D $6707-X$ & 9 & + & 5 & 4 & 13 & - & - & 3 & + & - & - \\
\hline D6707-W & 1 & - & 44 & 13 & 1 & - & - & 5 & - & - & - \\
\hline D6707-V & 2 & 8 & 12 & 51 & - & + & 1 & 1 & - & - & 7 \\
\hline D6707-S & - & 1 & 6 & 71 & 8 & + & - & - & - & - & 11 \\
\hline D6707-R & 1 & - & 11 & 11 & - & - & - & 3 & 2 & - & - \\
\hline D6707-P & 1 & 6 & 3 & 10 & 4 & - & - & 6 & - & - & + \\
\hline
\end{tabular}


TABLE 4

41. 42. 43. 46. 47. 50. 54. 57. 58. 59.60.61.

\begin{tabular}{|c|c|c|c|c|c|c|c|c|c|c|c|}
\hline D $6720-I$ & 7 & - & - & + & - & - & - & - & 7 & - & 1 \\
\hline D6 $720-\mathrm{H}$ & - & 1 & - & - & - & + & - & 15 & 8 & 1 & 2 \\
\hline D6 $720-G$ & 1 & + & - & - & - & + & - & - & 19 & 4 & 5 \\
\hline D6 $720-F$ & 1 & - & - & - & - & - & - & - & 5 & - & 2 \\
\hline D6 $720-E$ & - & - & 1 & - & - & - & - & 10 & - & - & 3 \\
\hline D6720-D & - & 1 & - & - & 2 & - & - & 5 & 3 & - & + \\
\hline D6720-C & - & - & - & - & 4 & - & - & - & - & - & - \\
\hline D6720-B & - & - & - & - & + & 1 & - & 6 & 8 & 1 & 54 \\
\hline D6720-A & - & - & + & - & 7 & + & 2 & 6 & 3 & + & 2 \\
\hline
\end{tabular}


TAB LE 4

41. 42. 43. 46. 47. 50. 54. 57. 58. 59.60.61.

\begin{tabular}{|c|c|c|c|c|c|c|c|c|c|c|c|}
\hline D6707-Z' & 1 & - & - & - & - & - & - & - & 1 & - & 1 \\
\hline D6707-Y & 6 & - & - & - & - & - & - & - & 8 & - & 25 \\
\hline D6707-X & + & - & - & 1 & - & - & - & - & 5 & - & + \\
\hline D6707-W & + & - & - & - & - & 1 & - & - & 18 & - & 21 \\
\hline D $6707-V$ & - & + & - & - & + & - & - & - & 9 & - & + \\
\hline D6707-s & - & - & - & 2 & - & - & - & 8 & 37 & + & 4 \\
\hline D6707-R & 2 & 1 & - & - & - & - & - & 9 & 2 & - & 1 \\
\hline D $6707-P$ & + & - & - & - & 2 & 2 & - & - & 3 & 13 & 8 \\
\hline
\end{tabular}


TABLE 4

62. 63. 64 .

D6720-I

D6720-H

D6720-G

D6720-F

D6720-E

D6720-D

D6720-C

D6720-B

D6720-A
2

$3-1$

$1 \quad-\quad 2$

$3-$

$8--$

$23-1$

$50-$

$20-10$

7

continued on p. 53

52 
TAB LE 4

62. 63.64.

$\begin{array}{llll}\text { D6707-Z' } & 1 & - & + \\ \text { D6707-Y } & 5 & - & - \\ \text { D6707-X } & 4 & + & - \\ \text { D6707-W } & 3 & - & - \\ \text { D6707-V } & 10 & - & - \\ \text { D6707-S } & 21 & - & - \\ \text { D6707-R } & 14 & - & 1 \\ \text { D6707-P } & 31 & - & 1\end{array}$




\title{
APPENDIX 2
}

Appendix 2 is a list of all palynomorph samples examined during the course of this investigation. All locality data are given in meters from two section boundaries. The stratigraphic position of surface samples is given in meters in relation to the major coals; sample position in cores is given as depth in meters relative to the ground surface.

$\begin{array}{lr}\text { USGS Paleobotany } & \text { Section/Locality } \\ \text { Locality No. } & \text { Sample data }\end{array}$

OR Creek Section

$20 \mathrm{mNL} 440 \mathrm{mEL}$, Sec. 30, T. 54 N., R. $77 \mathrm{~W}$. , Sheridan Co., Wy; Arvada Quadrangle

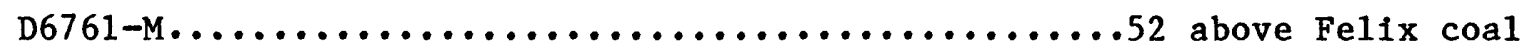

D6761-L........................................ "

D6761-K................................... "

D6761-J................................... *

D6761-I................................ "

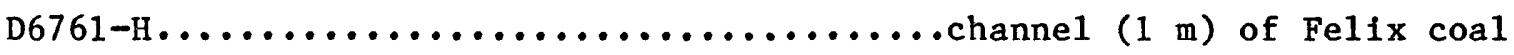

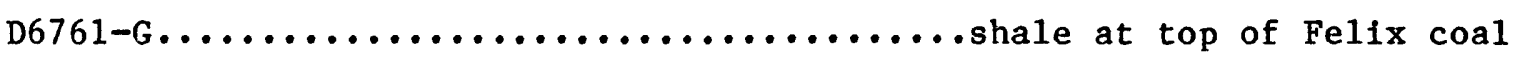

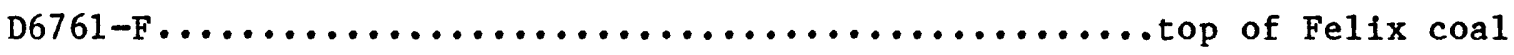

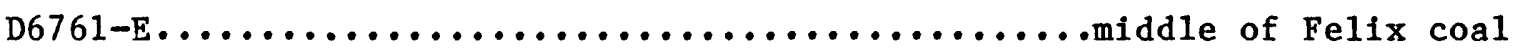

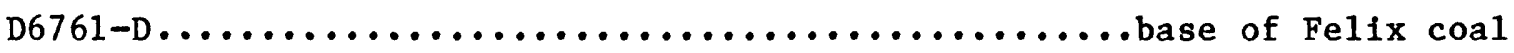

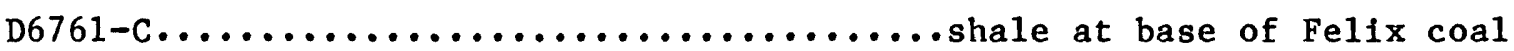

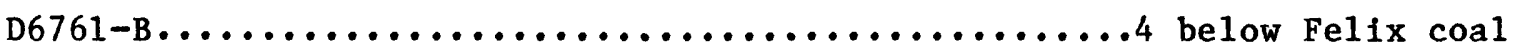

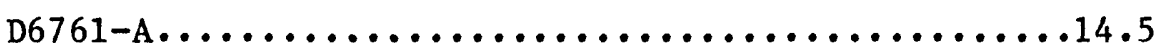

Core 1A

$10 \mathrm{mWL} 20 \mathrm{mSL}, \mathrm{Sec} .2, \mathrm{~T} .55 \mathrm{~N}$, , R. $77 \mathrm{~W}$., Sheridan Co. Wy; Cabin Creek SE Quadrangle

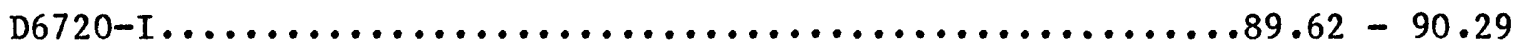

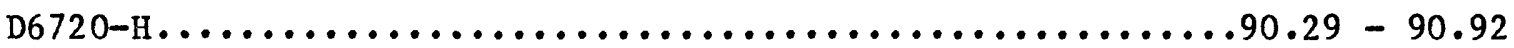




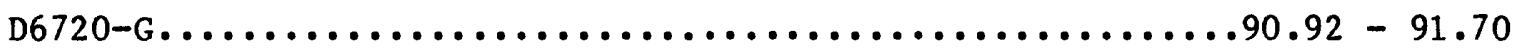

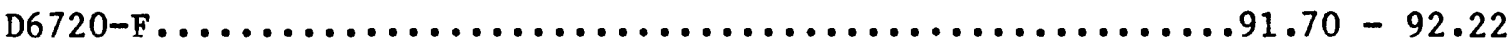

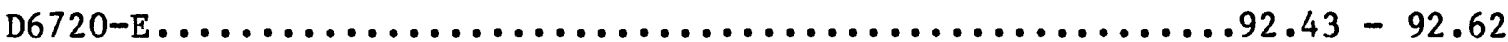

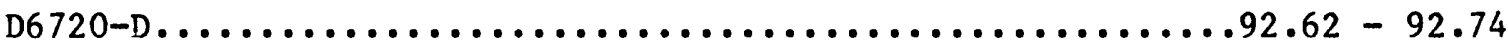

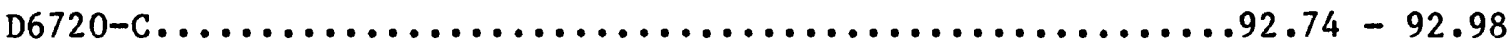

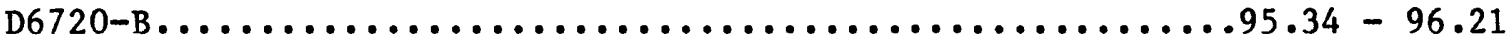

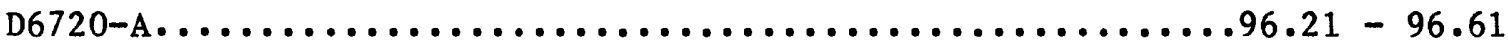

\section{Core 2A}

$780 \mathrm{mEL} 1140 \mathrm{mNL}$, Sec. 30, T. $55 \mathrm{~N}$., R. $77 \mathrm{~W}$.,

Sheridan Co., Wy; Cabin Creek SE Quadrangle

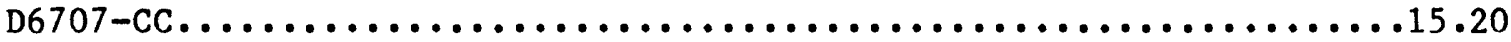

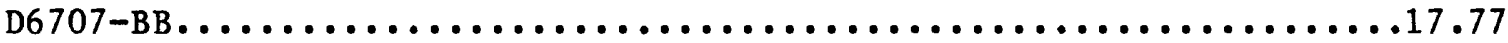

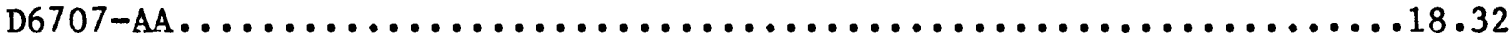

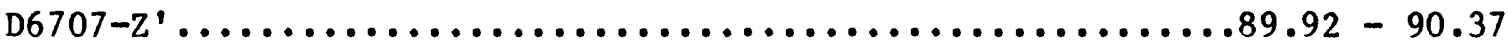

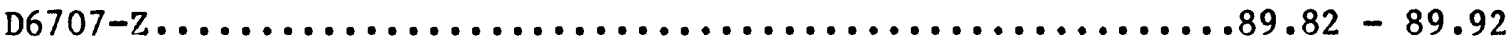

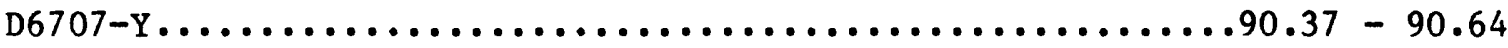

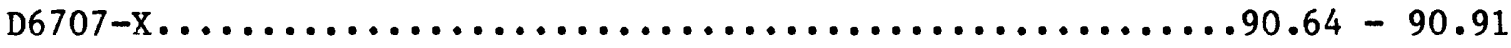

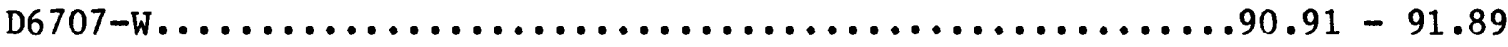

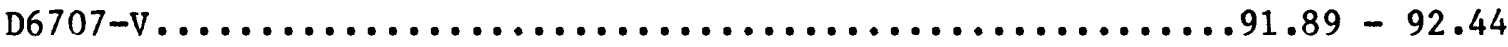

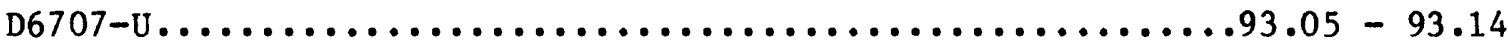

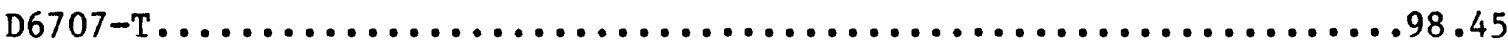

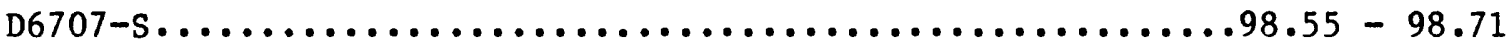

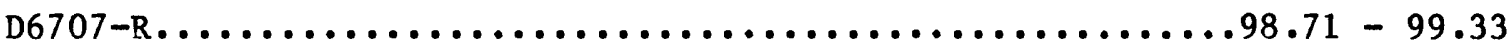

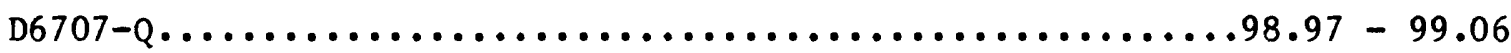

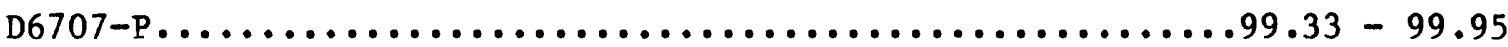

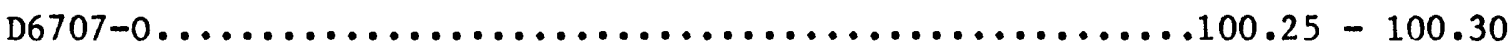

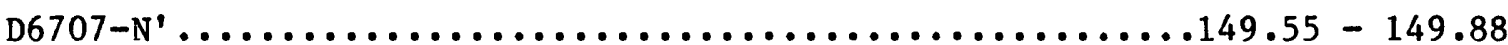

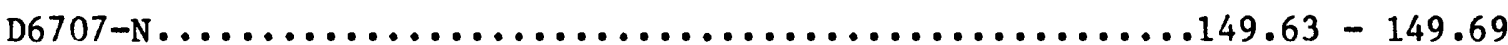

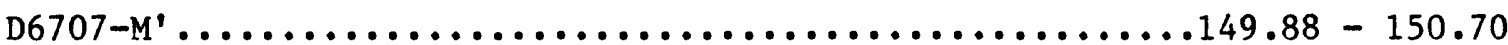

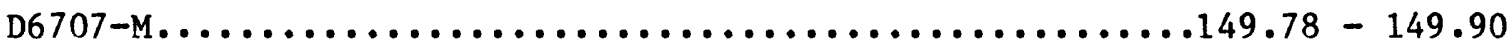

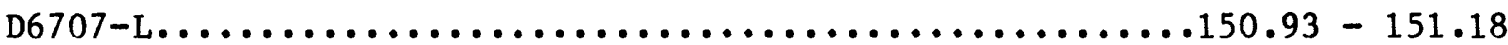

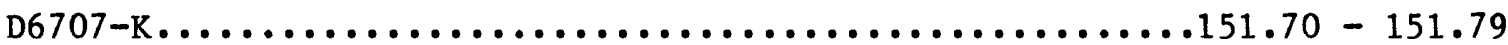

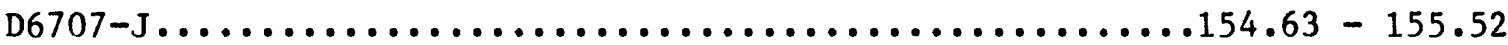


D6707-I.................................................. 154.08

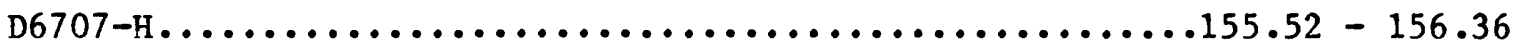

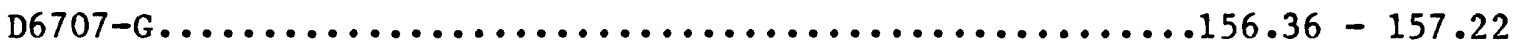

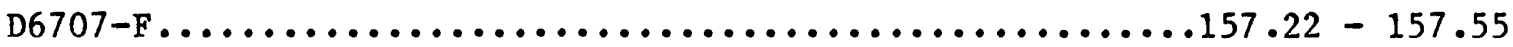

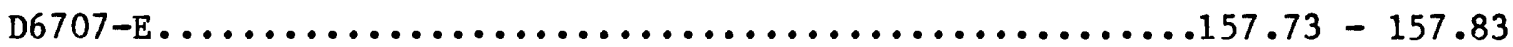

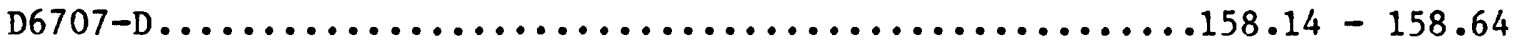

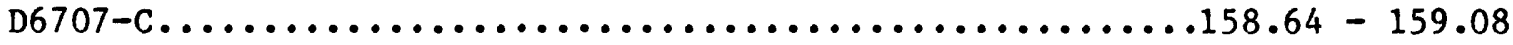

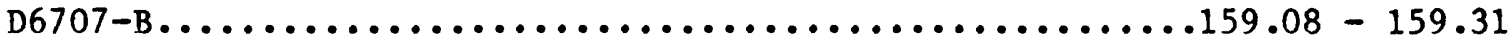

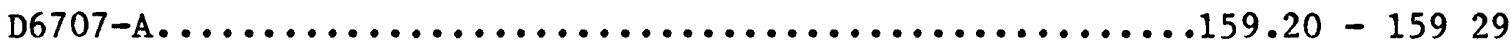

\section{Core 3A}

$20 \mathrm{mEL} 1220 \mathrm{mNL}$, Sec. 14, T. $56 \mathrm{~N}$., R. $77 \mathrm{~W}$.,

Sheridan Co., Wy; Cabin Creek SE Quadrangle

D6708-0................................................... 53.67 .60

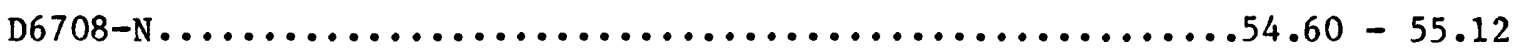

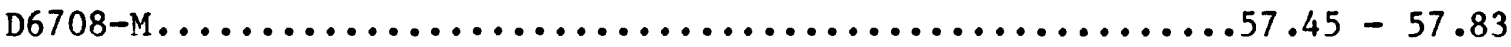

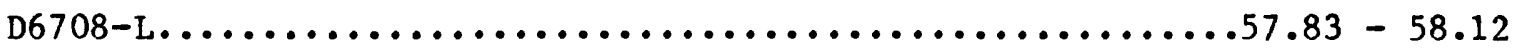

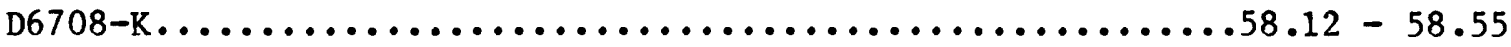

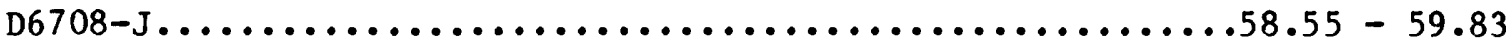

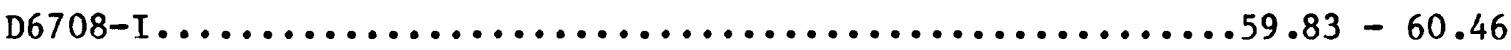

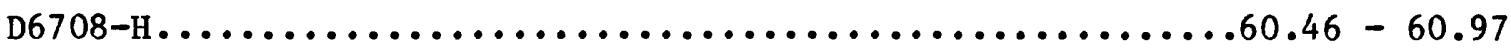

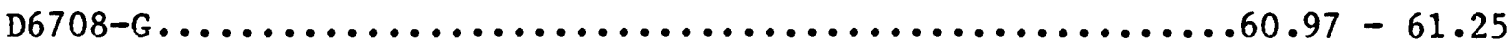

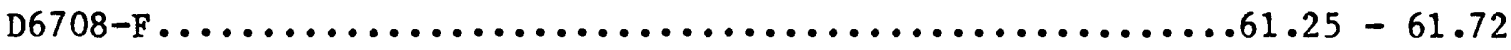

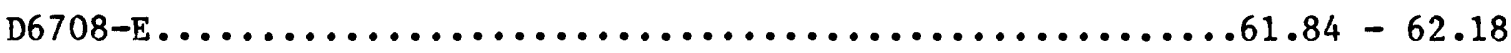

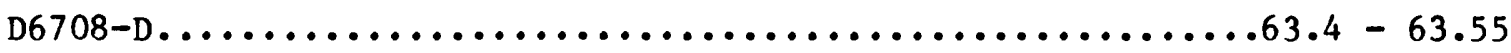

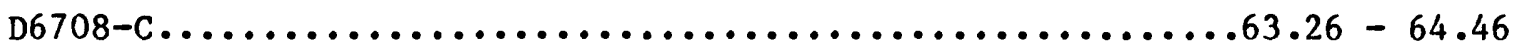

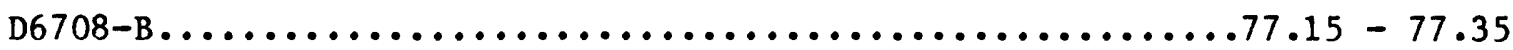

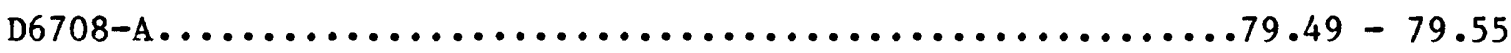




\section{Core 4A}

$1380 \mathrm{mNL} 1825 \mathrm{mEL}$, Sec. 31, T. 57 N., R. 76 W., Sheridan Co., Wy; Cabin Creek SE Quadrangle

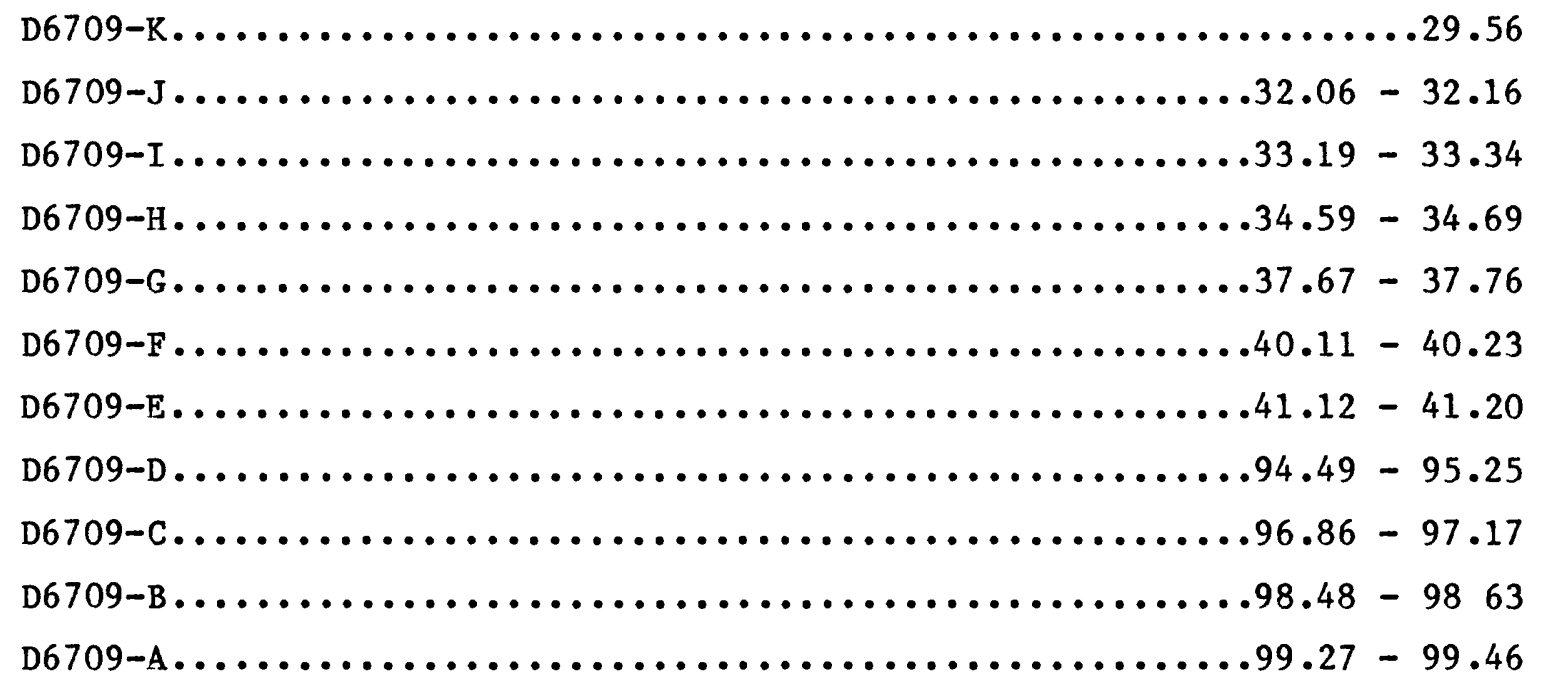

Core 80 AU-24

$1190 \mathrm{mNL} 1280 \mathrm{mEL}$, Sec. 11, T. 56 N., R. 77 W., Sheridan Co., Wy; Cabin Creek SE Quadrangle

D6710-E................................................ 26.08

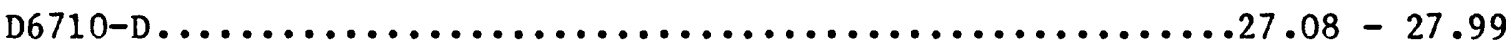

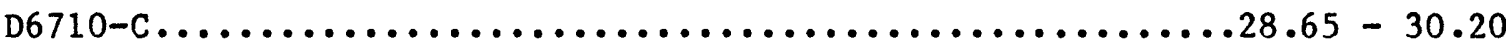

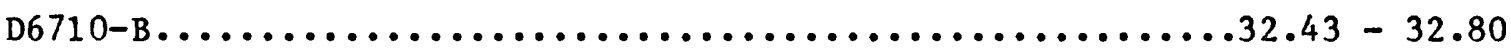

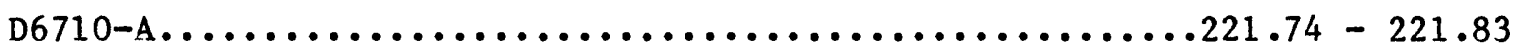

\section{Core 5A}

$110 \mathrm{mNL} 1450 \mathrm{mEL}$, Sec. 1, T. 8 S., R. $48 \mathrm{E}$., Powder River Co., Mt; Bloom Creek Quadrangle

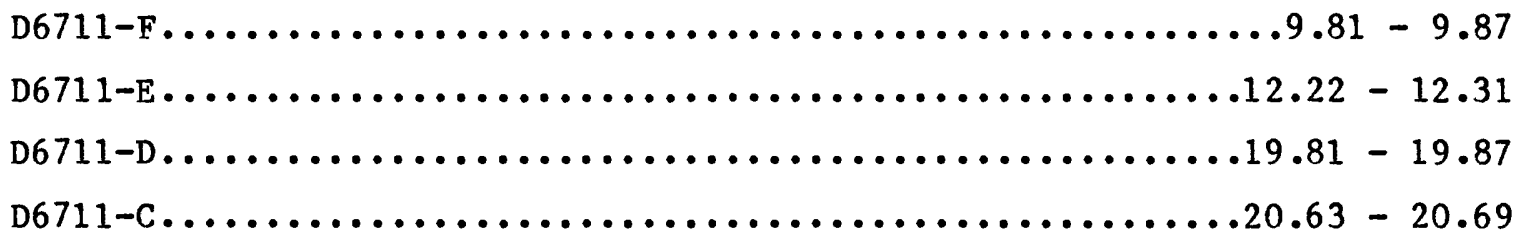




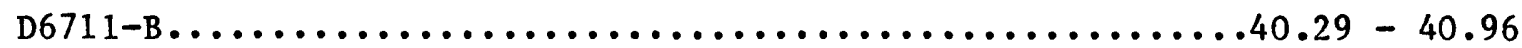

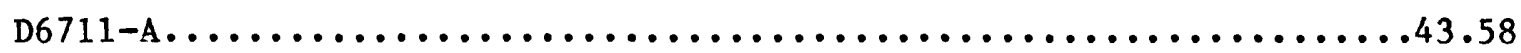

\section{Surface sections}

D6768..........'De11 coal..........120 mSL $515 \mathrm{mEL}$, Sec. 4, T. 3 S., R. 49 E., Powder River Co., Mt;

Leslie Creek Quadrangle

D6767.....16.1 below 0'Dell coal......55 mNL $620 \mathrm{mEL}$, Sec. 9, T. 3 S.,

R. 49 E., Powder River Co., Mt;

Leslie Creek Quadrangle

D6766-A \& B....Local 2 coal..........90 mSL $280 \mathrm{mEL}$, Sec. 30, T. 3 S., R. 49 E., Powder River Co., Mt;

Leslie Creek Quadrangle

D6765.......Mackin-Walker coal........510 mWL $560 \mathrm{mSL}$, Sec. 22, T. 3 S., R. 49 E., Powder River Co., Mt;

Leslie Creek Quadrangle

D6764...9.1 below Mackin-Walker coal...430 mWL $780 \mathrm{mSL}, \mathrm{Sec}$. 22, T. 3 S., R. 49 E., Powder River Co., Mt;

Leslie Creek Quadrangle

D6763....15.7 above Sawyer coal......420 mNL $625 \mathrm{mEL}$, Sec. 35, T. 3 S., R. 49 E., Powder River Co., Mt;

Leslie Creek Quadrangle

D6762.........Sawyer coal...........515 mEL $610 \mathrm{mNL}$, Sec. 35, T. 3 S., R. 49 E., Powder River Co., Mt; Leslie Creek Quadrangle 